\title{
Compact E-Cash with Practical and Complete Tracing
}

\author{
Bin Lian ${ }^{1,2^{*}}$, Gongliang Chen ${ }^{2}$, Jialin $\mathrm{Cui}^{1}$ and Dake $\mathrm{He}^{3}$ \\ ${ }^{1}$ Ningbo Institute of Technology, Zhejiang University, Ningbo, 315100 - China \\ ${ }^{2}$ School of Information Security Engineering, Shanghai Jiao Tong University, Shanghai, 200240 - China \\ ${ }^{3}$ School of information science and technology, Southwest Jiaotong University, Chengdu, 610031 - China \\ [e-mail: lianbin_a@163.com; chengl@sjtu.edu.cn; cuijl_jx@163.com; dkhe_scce@home.swjtu.edu.cn] \\ *Corresponding author: Bin Lian
}

Received August 14, 2018; revised October 18, 2018; accepted January 20, 2019;

published July 31, 2019

\begin{abstract}
E-cash has its merits comparing with other payment modes. However, there are two problems, which are how to achieve practical/complete tracing and how to achieve it in compact E-cash. First, the bank and the TTP (i.e., trusted third party) have different duties and powers in the reality. Therefore, double-spending tracing is bank's task, while unconditional tracing is TTP's task. In addition, it is desirable to provide lost-coin tracing before they are spent by anyone else. Second, compact E-cash is an efficient scheme, but tracing the coins from double-spender without TTP results in poor efficiency. To solve the problems, we present a compact E-cash scheme. For this purpose, we design an embedded structure of knowledge proof based on a new pseudorandom function and improve the computation complexity from $O(k)$ to $O(1)$. Double-spending tracing needs leaking dishonest users' secret knowledge, but preserving the anonymity of honest users needs zero-knowledge property, and our special knowledge proof achieves it with complete proofs. Moreover, the design is also useful for other applications, where both keeping zero-knowledge and leaking information are necessary.
\end{abstract}

Keywords: Compact E-cash, practical tracing, complete tracing, special knowledge proof, knowledge-leak, zero-knowledge proof

This research work is supported by Zhejiang Provincial Natural Science Foundation of China (No: LY17F020019, LY16F010019), National Natural Science Foundation of China (No: 61271220), National key research and development plan (No: 2017YFB0802505) and Natural Science Foundation of Ningbo (No: 2016A610212). 


\section{Introduction}

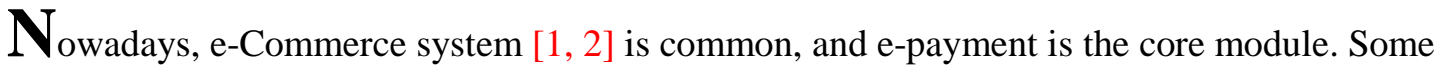
e-payments guarantee the anonymity of users, but sometimes it is abused for crimes. Over the past years, quite some research effort has been put in design of E-cash [3] based on blind signature $[4,5]$ or knowledge signature [6, 7]. To prevent user from abusing anonymity, the tracing function is necessary. Fig. 1 shows a typical E-cash model.

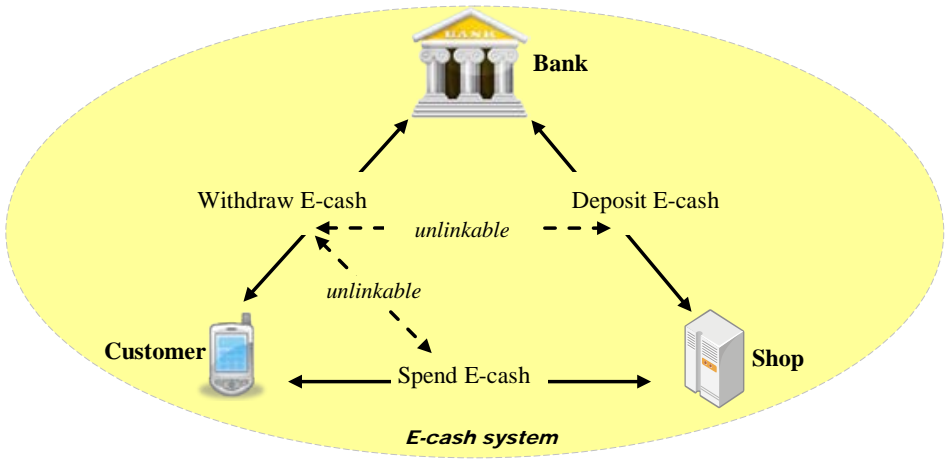

Fig. 1. Typical E-cash model

In general, an E-cash system has the following properties:

- Anonymity: No one can identify the spender or link the spending behaviors.

- Unreusability: The owner of e-cash will be identified if he spends one coin twice.

- Unforgeability: No one except the bank can generate valid e-cash.

- Offline mode: There is no the third party participating in the payment process.

- Environmental independence: System security depends on the cryptographic scheme.

Some interesting E-cash schemes such as divisible E-cash, transferable E-cash and changeable denomination E-cash, etc., are put forward. And compact E-cash [8] is the important scheme. In such system, user withdraws $2^{l}$ e-coins by performing the withdrawal protocol one time and stores the coins in $O(l)$ bits.

\section{Related Work}

Our goal is to construct a compact E-cash system with practical and complete tracing, so we present two parts of the related work.

Part I: the Practical tracing and the Complete tracing

"Practical" means that different tracings should be provided by the appropriate entity, and "Complete" means that the tracings should meet the demand from real-world applications.

To prevent customer from reusing a coin, double-spending tracing is the basic function. Since it is related to the bank's business, double-spending tracing should be performed by bank.

Sometimes, the anonymity of users may provide the convenience for crimes, such as money laundering and blackmailing. Therefore, S. von Solms and D. Naccache [9] suggested that the anonymity of users should be revoked when necessary, and then [10] and [11] put forward a new E-cash scheme, i.e., fair E-cash. In such a system, the user remains anonymous if he honestly spends the legal e-coins, but if crimes occur so that the related transaction is illegal, the e-coins and the owner can be traced unconditionally. Here "unconditionally" means that the coins and the owner can be traced when the user does not double-spend coins. 
In reality, the authorities are usually the trusted third parties, who have the power to perform unconditional tracing only when the E-cash is involved in crimes.

Therefore, it is not practical that the TTP is indispensable to double-spending tracing $[1,10$, $12,13,14,15,16,17,18]$. But the tracing function is not complete if the system does not provide unconditional tracing $[2,8,19,20,21,22,23,24,25,26,27]$.

In our opinion, for crime prevention, the unconditional tracing is necessary, however, the existence of TTP unavoidably threatens the anonymity of honest users. So it is reasonable that the system can be independent of TTP, but at the same time, the scheme can easily be slightly modified to select the available tracings depending on the actual circumstances.

In addition, if the user loses E-cash, he should find some way to get his money back if the lost E-cash cannot be spent by anyone. And it could be achieved if bank provides lost-coin tracing (in the validity period of coins), which is ignored by almost all schemes.

There are $2^{l}$ coins in the customer's wallet in compact E-cash system. So in such system, double-spending tracing and unconditional tracing should include coin-tracing. Table 1 shows the property of the practical and complete tracing.

Table 1. The property of the practical and complete tracing

\begin{tabular}{ccc|cc}
\multirow{2}{*}{ Tacing types } & \multicolumn{1}{c}{ Double-spending tracing } & Unconditional tracing & Loss tracing \\
\cline { 2 - 4 } Provider & tracing spender & tracing coins & tracing owner tracing coins & tracing coins \\
\hline Condition & Bank & TTP & Bank \\
\hline Module & Double-spending & None (only in crime cases) & Valid loss-register for unspent coins \\
\hline
\end{tabular}

In fact, the "practical" and "complete" tracing is not easy for existing schemes. Moreover, the recoverable E-cash scheme is presented in $[20,28,29]$ for sloving the coin-lost problem, but there were some unsolved problems. In our [30], we clarify all there problems and provide a solution. But to achieve the practical and complete tracing in compact E-cash, there is a new problem.

Part II: the Efficiency problem in compact E-cash

The significant contribution of compact E-cash [8] is that the computation complexity of withdrawing $2^{l}$ coins is $O(1)$, and the storage space of the $2^{l}$ e-coins is just $O(l)$ bits, while before it is proposed, the E-cash schemes have to withdraw $2^{l}$ coins or store them with $O\left(2^{l}\right)$ complexity. Then some other interesting compact E-cash schemes [21, 23, 31] are designed based on different cryptographic techniques. But good efficiency, which is the very important for compact E-cash, does not attract enough attention in the designing. After all, achieving efficient system is the main goal of compact E-cash.

The main efficiency problem is that tracing coins without TTP results in poor efficiency [8, 21, 23, 31]. When the user double-spends E-cash, tracing him is necessary. However, tracing his coins is very important, since the doulbe-spender has at least $2^{l}$ coins so that he can cheat more times if system cannot trace his remaining coins. As we discussed, double-spending tracing is the bank's task, not the TTP's. Obviously, if TTP performs every tracing, it seriously threatens the anonymity of honest users. However, when double-spending, it is not easy for existing schemes that bank traces double-spender's coins without TTP.

[21, 23] and the system 1 of [8] only provide the double-spender tracing. The system 2 of [8] and [31] provide coin-tracing. However, when adding this coin-tracing without TTP, their systems [8, 31] become inefficient. For example, when the system 2 of [8] adds coin-tracing, the inefficiency is pronounced — in the system 1, the withdrawal uses 12 multi-based exponentiations, while in the system 2, it uses 810 multi-based exponentiations and 300 
bilinear pairings. At the same time, in the system1, spending one coin uses 40 multi-based exponentiations, while in the system 2, it uses 778 multi-based exponentiations.

In existing compact E-cash schemes [8, 21, 23, 31], there are some difficulties in designing coin-tracing without TTP for doulbe-spending. And we will clarify it. All compact E-cash schemes are constructed based on zero-knowledge proof. In short, when spending a coin from the compact E-cash wallet, the customer shows the shop a pseudorandom function with the wallet parameters and proves the pseudorandom function is constructed correctly using zero-knowledge proof. So when the customer doulbe-spends a coin, the bank needs the wallet parameters as the input of the pseudorandom function so as to trace the remaining coins from the double-spender. But without a TTP, for recovering the secret wallet parameters, [8] uses the verifiable encryption and [31] uses the accumulator. Unfortunately, these cryptographic techniques result in poor efficiency.

Tacing without TTP implies that the information of secret parameters is leaked when double-spending. But for the anonymity of honest customers, the knowledge of secret parameters is proven using zero-knowledge proof. Therefore, to solve the problem of recovering secret parameter, our idea is reconstructing the zero-knowledge proof [32]. And the new zero-knowledge proof can leak the information of the proven parameters when it is used twice to prove the same knowledge (double-spending), but it has the perfect zero-knowledge property when it is used once (normal-spending). That is to say, it guarantees the anonymity in normal case and revokes the anonymity in abnormal case.

To the best of our knowledge, when the customer double-spends a coin, the efficiency problem caused by tacing coins without TTP had not been solved in existing compact E-cash schemes. And we solve it — achieving coin-tracing without TTP, our computation complexity of withdrawing E-cash is $\boldsymbol{O ( 1 )}$, while it is $\boldsymbol{O (} \boldsymbol{k})$ in [8] and it is $\boldsymbol{O}\left(\mathbf{2}^{2 n}\right)$ in [31].

Our main contributions:

$\diamond \quad$ We present a compact E-cash scheme with practical and complete tracing.

$\diamond$ We provide a solution to the efficiency problem in compact E-cash system when tracing double-spender's coins without TTP.

$\diamond \quad$ The new knowledge proof is zero-knowledge to verifier in normal-spending, while it leaks the proven knowledge in double-spending.

In the following parts, Section 3 provides preliminaries. Section 4 presents the system model. In section 5, we describe the tracing mode. Section 6 provides the details of the proposed scheme. The key security proofs are presented in section 7 . And in section 8 , we compare the system efficiency of schemes. Finally, the conclusion is provided in section 9.

\section{Preliminaries}

\subsection{Assumptions}

Assumption 1 (S-RSA Assumption) [33]. Let $n=p q$ be an RSA-like modulus and $z \in \mathrm{Z}_{n}^{*}$. It is hard to compute $u \in \mathrm{Z}_{n}^{*}$ and integer $e>1$ such that $z \equiv u^{e}(\bmod n)$.

Assumption 2 (DDH Assumption) [34]. Let $G=\langle g\rangle$ be a cyclic group generated by $g$ of order $u=\# G$ with $\left\lceil\log _{2}(u)\right\rceil=l_{G}$. Given $\left(g, g^{x}, g^{y}, g^{z}\right) \in G^{4}$, it is hard to decide whether $g^{z}$ and $g^{x y}$ are equal.

Assumption 3 (q-DDHI Assumption) [8]. Let $G=\langle g\rangle$ be a cyclic group generated by g. Given the elements $\left(g, g^{x}, \ldots, g^{\left(x^{q}\right)}\right) \in\left(G^{*}\right)^{q+1}$, it is still hard to decide whether $g^{1 / x}$ and a random element in $G$ are equal.

The $(\boldsymbol{t}, \boldsymbol{q}, \boldsymbol{\varepsilon})$-DDHI assumption means that there is no $\boldsymbol{t}$-time algorithm which has the 
advantage at least $\boldsymbol{\varepsilon}$ to break the $\boldsymbol{q}$-DDHI assumption.

\subsection{Zero-knowledge Proof [35, 36]}

Definition 1. Let $A=\{A(x)\}_{x \in L}$ and $B=\{B(x)\}_{x \in L}$ be two ensembles of variables indexed by strings $x \in L$, where $L \in\{0,1\}^{*}$. A and $B$ are statistically indistinguishable, if for any polynomial $p($.$) and any x \in L$ it holds that $\Sigma_{t \in\{0,1\} *}|\operatorname{Prob}(A(x)=t)-\operatorname{Prob}(B(x)=t)|<1 / p(|x|)$.

Definition 2. Protocol $(P, V)$ is statistical zero-knowledge, if $\{[P, V](x)\}_{x \in L}$ and $\left\{S_{[P, V]}(x)\right\}_{x \in L}$ are statistically indistinguishable, where $V$ can be any probabilistic polynomial-time verifier and $S_{[P, V]}$ is a probabilistic polynomial-time simulator which can simulate the protocol $(P, V)$. Definition 3. $(c, s) \in\{0,1\}^{k} \times \pm\{0,1\}^{\varepsilon\left(l_{G}+k\right)+1}$ satisfying $c=H\left(y\|g\| g^{s} y^{c} \| m\right)$ is a signature of knowledge (SPK) on the message $m$, which uses the knowledge of discrete logarithm of $y$ to the base $g$. And $\operatorname{SPK}\left(\alpha: y=g^{\alpha}\right)(m)$ denotes it.

Using the knowledge of discrete logarithm, i.e., $x=\log _{g} y$, $\boldsymbol{S P K}\left(\alpha: y=g^{\alpha}\right)(m)$ is computed as follows. After choosing $r \in \pm\{0,1\}^{\varepsilon\left(l_{G}+k\right)}$, the signer computes $c=H\left(y\|g\| g^{r} \| m\right.$ ) (i.e., challenge) and $s=r-c X$ (i.e., challenge-response), and $g^{r}$ is the commitment to prove that the signer knows $x=\log _{g} y$. Here $H():\{0,1\}^{*} \rightarrow\{0,1\}^{k}$ denotes a hash function. The interactive protocol of $\boldsymbol{S P K}$ performed by prover and verifier is the zero-knowledge proof of the knowledge of $x=\log _{g} y$ provided by prover, which is denoted by $\boldsymbol{P K}($ ) [8].

\subsection{Pseudorandom Function [37]}

Generating Key: Choose the secret key $S K \in_{R} Z_{p}{ }^{*}$, and the public key $P K=g^{S K}$.

Verifiable Random Function: $F_{S K}(x)=e(g, g)^{1 /(x+S K)}$ is a verifiable random function and $p_{S K}(x)=g^{1 /(x+S K)}$ is the proof of correctness of it.

Verification: Verify $e\left(g^{x} \cdot P K, p_{S K}(x)\right)=e(g, g)$ and $F_{S K}(x)=e\left(g, p_{S K}(x)\right)$. If it is true, $F_{S K}(x)$ is proven to be generated correctly.

So $F_{S K}(x)=h^{1 /(x+S K)}$ is viewed as a pseudorandom function ( $\left.\underline{\mathbf{P R F}}\right)$ [37]. $F_{S K}(x)$ is an $\left(s^{\prime}(k)\right.$, $\left.\varepsilon^{\prime}(k)\right)$ secure PRF if no one can break the pseudo randomness property with $\varepsilon^{\prime}(k)$ advantage in $s^{\prime}(k)$ time.

\section{Security Model of the E-cash System}

\subsection{Syntax}

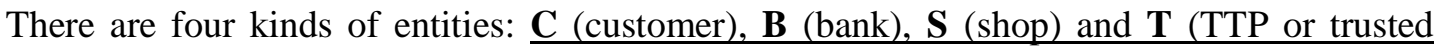
authorities). There are the polynomial time algorithms or protocols: B/T/C Setup, Withdraw, Pay, Deposit, UnconditionallyTrace, LossCoinTrace, DoubleSpendTrace. P(E1 $\left(x_{1}\right)$, E2 $\left.\left(x_{2}\right)\right)$ denotes a protocol between E1 and E2, and E1's input is $x_{1}$ and E2's is $x_{2}$.

$\diamond \mathrm{B} / \mathrm{T} / \mathrm{C}$ Setup(params). The algorithm outputs $\mathrm{B} / \mathrm{T} / \mathrm{C}$ 's private/public key pair $\left(P K_{B}, S K_{B}\right) /\left(P K_{T}, S K_{T}\right) /\left(P K_{C}, S K_{C}\right)$, here $\mathrm{C}$ includes $\mathrm{S}$.

$\diamond \quad$ Withdraw $\left(\mathrm{C}\left(S K_{C}, P K_{B}\right), \mathrm{B}\left(P K_{C}, S K_{B}\right)\right)$. It allows $\mathrm{C}$ to withdraw a certain amount of E-cash. C receives $X$ (E-cash), i.e. an identifier $I$ and a proof of validity $\Pi$, or one error message $\perp$. B receives the view of the protocol (we call this $V_{P K C}$ ) or one error message $\perp$ if $V_{P K C}$ is not proven from $C$ with $P K_{C}$.

$\diamond \quad \operatorname{Spend}\left(\mathrm{C}\left(X, P K_{B}, S K_{C}\right), \mathrm{S}\left(P K_{S}\right)\right)$. It allows $\mathrm{C}$ to pay e-coin from $X$ to $\mathrm{S}$. $\mathrm{S}$ receives the proof $\pi$ of payment with aux (auxiliary information) or one error message $\perp$ if $\pi$ is invalid. C receives the updated wallet $X^{\prime}$ if the payment is accepted by S. 
$\diamond \quad \operatorname{Deposit}\left(\mathrm{S}\left(P K_{S}, \pi, a u x\right), \mathrm{B}\left(P K_{B}\right)\right)$. It allows $\mathrm{S}$ to send $\left(P K_{S}, \pi, a u x\right)$ to $\mathrm{B}$ for deposit. After verifying $\left(P K_{S}, \pi, a u x\right)$, B adds $(\pi, a u x)$ to spent records. But $\mathrm{B}$ outputs one error message $\perp$ if $\left(P K_{S}, \pi, a u x\right)$ is invalid or B executes the DoubleSpendTrace algorithm if the coin is double-spent.

$\diamond$ UnconditionallyTrace $\left(\mathrm{B}\left(V_{P K C}, \pi, a u x\right), \mathrm{T}\left(S K_{T}\right)\right)$. It is an algorithm which allows $\mathrm{T}$ to trace any e-coin from $X$ and any owner when the transaction is involved in crimes. $\mathrm{T}$ outputs particular e-coin tracing information $I_{e-c o i n}$, owner tracing information $I_{o w n e r}$ and proof $P_{1}$ which proves the connection between the e-coin from $X$ and $\mathrm{C}$, but if the input is illegal, T outputs an error message $\perp$.

$\diamond$ LossCoinTrace $\left(\mathrm{C}\left(S K_{C}\right), \mathrm{B}\left(P K_{C}, V_{P K C}\right)\right)$. It allows $\mathrm{C}$ to register his lost coin/wallet in system. B outputs loss tracing information $I_{\text {loss }}$ and a proof $P_{2}$ which proves the validity of the loss register without $\mathrm{T}$, but if the proof of owning the lost-coin/wallet can't be provided, B outputs an error message $\perp$; If the lost coin was spent, B outputs the spent proof $P_{\text {spent. }}$.

$\diamond$ DoubleSpendTrace $\left(\pi_{1}, \pi_{2}\right)$. With double-spending proofs related to one coin, B executes the algorithm and outputs $P K_{C}$ of the double-spender, the tracing information $I_{X}$ of the coins from $X$ and the proof $P_{3}$ which proves that $C$ with $P K_{C}$ is the double-spender and the traced coins with $I_{X}$ is owned by the double-spender, but if $\pi_{1}=\pi_{2}$, the algorithm outputs an error message $\perp$.

\subsection{Security Definitions}

- Balance. In the Withdraw protocol, Withdraw $\mathrm{w}_{\mathrm{m}}$ is the middle of the protocol. $m_{1}$ is the first message sent by $\mathrm{C}$ and $b_{1}$ is B's state information when $m_{1}$ is received. The balance property indicates that:

- There are the efficiently decidable language $l_{S}$ and the extractor $\varepsilon_{\text {Withrawm,ls }}^{X-Y(A)}$ (params, auxext, $\left.P K_{C}, m_{1}, b_{1}\right)[8]$ such that for all $b_{1}$ and $m_{1}$, which extracts $w=\left(\Theta_{1}, \ldots, \Theta_{n}, \sec _{w}\right)$ such that $\left(m_{1}, b_{1}, w\right) \in l_{S}$ whenever the probability that B accepts in the Withdraw ${ }_{\mathrm{m}}$ part of the protocol is non-negligible, where $\Theta$ is the serial number of E-cash (e-coin). In the case, the extractor outputs $\left(m_{1}, b_{1}, w\right) \in l_{S}$.

- With (params, $P K_{B}$ ), the adversary $\mathcal{A}$ plays the game as follows: $\mathcal{A}$ performs Withdraw/Deposit protocols with B polynomial times. (A simulates running Spend protocol with itself.) $\left(m_{1, i}, b_{1, i}, w_{i}\right) \in l_{S}$ is the output of $\varepsilon_{\text {Withrawm }}^{X-(A)}$ (params, auxext, $P K_{C i}, m_{1, i}$, $\left.b_{1, i}\right)$ if the $i$ th Withdraw protocol is successful, where $w_{i}=\left(\Theta_{i, 1}, \ldots, \Theta_{i, n}\right.$, $\left.\sec _{w i}\right)$ are $2^{l}$ serial numbers belonging to $P K_{C i}$. $A_{f}=\left\{\Theta_{i, j} \mid 1 \leq i \leq f, 1 \leq j \leq n\right\}$ is the set of all serial numbers after performing Withdraw protocol $f$ times. $\mathcal{A}$ wins this game if for some $f$, B accepts a coin with one $\Theta \notin A_{f}$ in Deposit protocol. The secure E-cash scheme requires that no probabilistic polynomial-time (PPT) $A$ can win the game with non-negligible probability.

- Complete-Tracing. It guarantees that no PPT $A$ has a non-negligible probability of winning the following game:

On input (params, $P K_{B}$ ), $\mathcal{A}$ performs Withdraw/Deposit protocols with B polynomial times. ( $A$ A also simulates running Spend protocol with itself.) $A_{i}$ be the set of serial numbers which belong to $\mathrm{C}_{i}$ with $P K_{C i}$. $A$ wins the game if any of the following cases occurs.

- $\quad$ On input $\left(\mathrm{B}\left(V_{P K C}, \pi, a u x\right), \mathrm{T}\left(S K_{T}\right)\right)$, the algorithm UnconditionallyTrace can not output e-coin tracing information $I_{e-c o i n-i}$, owner tracing information $I_{\text {owner-i }}$ or proof $P_{1 i}$ which proves the coins from $X_{i}$ owned by $C_{i}$; 
- On input $\left(\mathrm{C}_{i}\left(S K_{C i}\right), \mathrm{B}\left(P K_{C i}, V_{P K C}\right)\right)$, the protocol LossCoinTrace can not output loss tracing information $I_{\text {loss- } i}$ or a proof $P_{2 i}$ which proves the validity of the loss register;

- On input $\left(\pi_{1}, \pi_{2}\right)$ of the same coin, in some Deposit protocol, B accepts the same coin spent twice with serial number $\Theta_{i, j} \in A_{i}$ twice, i.e., B accepts $\left(\Theta_{i, j}, \pi_{1}, j\right)$ and $\left(\Theta_{i, j}, \pi_{2}, j\right)$ and cannot find the double-spending behavior;

- On input $\left(\pi_{1}, \pi_{2}\right)$ of the same coin, DoubleSpendTrace cannot output double-spender's public key or the related information of the coins in $X_{i}$ from double-spender for tracing;

- On input $\left(\pi_{1}, \pi_{2}\right)$ of the same coin, DoubleSpendTrace cannot output the proof $P_{3 i}$, which proves that $C_{i}$ with $P K_{C i}$ is double-spender or all the traced coins are owned by the double-spender $\mathrm{C}_{i}$.

- Anonymity of customer. $A$ generates B's pulic key $P K_{B}$ and then plays the following games arbitrarily:

Game $\mathrm{R}_{1}$

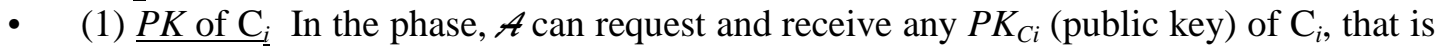
generated in Setup phase.

- (2) Withdrawing with $\mathrm{C}_{i} A$ performs Withdraw protocol with $\mathrm{C}_{i}$ : Withdraw $\left(\mathrm{C}\left(S K_{C i}, P K_{B}\right.\right.$, $n), A($ state, $n)$ ); $X_{j}$ is $C_{i}$ 's output after the $j$ 'th withdrawing, and $X_{j}$ could be the error message, e.g., $X_{j}$ is invalid.

- (3) Spending from $X_{i} \not A$ performs Spend protocol with $\mathrm{C}_{i}$ if $X_{j}$ is valid: $\operatorname{Spend}\left(\mathrm{C}\left(X_{j}, P K_{B}\right.\right.$, $\left.S K_{C i}\right), \mathcal{A}$ (state)). And $\mathcal{A}$ cannot request $\mathrm{C}_{i}$ to spend the same coin more than once.

$\underline{\text { Game } \mathrm{R}_{2}}$ The phases (1) and (2) are the same as them in Game $\mathrm{R}_{1}$, but in the phase (3), $\mathcal{A}$ performs Spend protocol with a simulator $S^{X-Y(A)}$ (params, auxsim, $P K_{B}$ ).

Without the knowledge of $x$ about Game $\mathrm{R}_{\underline{x}}$, $A$ guesses $x^{\prime}$ of Game $\mathrm{R}_{\underline{x}^{\prime}}$, where $x, x^{\prime} \in\{0,1\}$. Anonymity of customer means that $\delta=\left(\operatorname{Prob}\left(x^{\prime}=x\right)-1 / 2\right)$ is negligible for the PPT $\mathcal{A}$.

- Strong Exculpability. $A$ has the knowledge of secret key $S K_{B}$ and $S K_{C}$ of collusive $C$ and can act as $\mathrm{B}$ or $\mathrm{S}$ or collusive $\mathrm{C}$ in sytem protocols. $\mathcal{A}$ can choose any honest $\mathrm{C}_{i}$ with public key $P K_{C i}$ and perform system protocols with him arbitrarily. If any of the following cases occurs, $\mathcal{A}$ wins this game.

- $\quad$ Case $_{1}$ : If $\mathrm{C}_{i}$ is honest, i.e., $\mathrm{C}_{i}$ never double-spends one coin, DoubleSpendTrace algorithm outputs $\left(P_{3 i}, P K_{C i}, I_{X i}\right)$;

- Case $_{2}$ : If $C_{i}$ is honest, $\mathscr{A}$ gets some valid spending proof $\left(\pi_{i}, a u x_{i}, \Theta_{i}\right)$ where $\Theta_{i}$ is the serial number of some coin, but $\mathrm{C}_{i}$ has not spent the related coin;

- $\quad$ Case $_{3}$ : If $C_{i}$ spends one coin twice, $\mathscr{A}$ gets $\left(\pi_{1 i}, a u x_{1 i}, \Theta_{d i}\right)$ and $\left(\pi_{2 i}, a u x_{2 i}, \Theta_{d i}\right)$ respectively. Then $\left(P_{3 i}, P K_{C i}, I_{X i}\right)$ is the output of DoubleSpendTrace algorithm on input $\left(\pi_{1 i}, \pi_{2 i}\right)$. Then DoubleSpendTrace algorithm outputs $\left(P_{3 i}{ }^{\prime}, P K_{C i}, I_{X i}, \Theta_{d i}^{\prime}\right)$, but $C_{i}$ does not spend the coin with $\Theta_{d i}^{\prime}$ twice;

- $\quad$ Case $_{4}$ : If $C_{i}$ spends one coin twice, $\not$ gets $\left(\pi_{1 i}, a u x_{1 i}, \Theta_{d i}\right)$ and $\left(\pi_{2 i}, a u x_{2 i}, \Theta_{d i}\right)$ respectively. Then $\left(P_{3 i}, P K_{C i}, I_{X i}\right)$ is the output of DoubleSpendTrace algorithm on input $\left(\pi_{1 i}, \pi_{2 i}\right)$. Then $A$ gets some valid spending proof $\left(\pi_{i}^{\prime}\right.$, aux $\left.{ }_{i}^{\prime}, \Theta_{i}^{\prime}\right)$, but $C_{i}$ has not spent the related coin.

Strong Exculpability means the probability that the PPT $\mathcal{A}$ wins the above game is negligible.

\section{Tracing Mode of the Proposed Compact E-cash Scheme}

In the proposed scheme, only when crimes occur, TTP has the power to execute unconditional 
coin-tracing (according to the information from withdrawal protocol provided by bank) and unconditional owner-tracing (according to the information from deposit provided by shop). When customer registers for his lost coins, bank executes the lost-coin tracing without TTP. When customer spends a coin twice, bank executes the double-spender/coin tracing without TTP. From Fig. 2, we can see that the system with the basic tracing functions can be independent of TTP, that is to say, unconditional tracing is optional in our system.

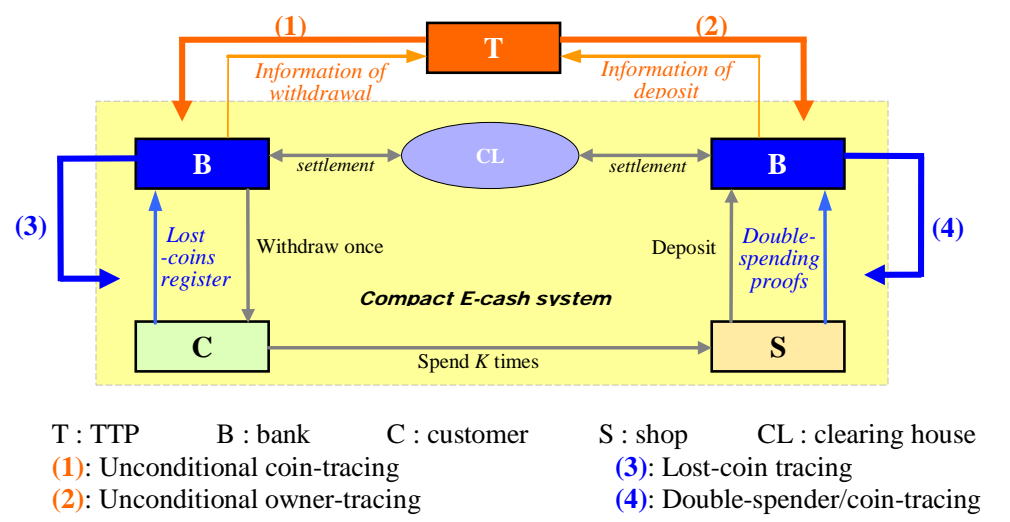

Fig. 2. Compact E-cash system providing complete and practical tracing

\section{Compact E-cash with Practical and Complete Tracing}

\subsection{Overview of the Proposed Scheme}

\section{Withdrawal Protocol}

$\mathrm{C}$ and $\mathrm{B}$ generate the wallet parameters $\left(e_{1}, e_{2}, x\right)$ which are used to generate $2^{l}$ coins, and $\mathrm{B}$ signs them using CL signature [38]. Then to achieve loss tracing and unconditional tracing, $\mathrm{C}$ provides two ElGamal encryptions_— ElGamal ${ }_{\mathrm{PKc}}\left(g^{e_{2}}\right)$ encrypts $g^{e_{2}}$ using C's public key $\mathrm{PK}_{\mathrm{C}}$ and ElGamal $\mathrm{PK}_{\mathrm{T}}\left(g^{e_{2}}\right)$ encrypts $g^{e_{2}}$ using T's public key $\mathrm{PK}_{\mathrm{T}}$. Also, C provides the knowledge proof $(S P K)$ to prove the encryptions are generated correctly.

\section{$\diamond$ Payment Protocol}

C performs the protocol with S to spend one of coins from C's wallet.

(1) To prove the validity of coin, C provides the zero-knowledge proof of $\left(e_{1}, e_{2}, x\right)$ to prove that the coin spent in this protocol is from some signed wallet.

(2) To achieve loss tracing and unconditional tracing, C computes $T_{2}=g^{H(J \| r) e_{2}}\left(\bmod n_{\mathrm{T}}\right)$ with the random input $r$, where $n_{\mathrm{T}}$ is RSA modulus and $\mathrm{T}$ has its factor knowledge, integer $J \in\left[0,2^{l}-1\right]$. C also provides the zero-knowledge proof to prove that $T_{2}$ is generated correctly.

(3) To prevent double-spending, $C$ provides the serial number of coin, i.e., $\Theta=\operatorname{PRF}\left(e_{2}, J\right)$, where $\operatorname{PRF}\left(\right.$ ) is a pseudorandom function with the secret seed $e_{2}$ and the public input $J$. The $\Theta$ records the spent coins for B and the integer $J \in\left[0,2^{l}-1\right]$ records the spent coins for C. So C's wallet contains $2^{l}$ coins. Also, C provides the zero-knowledge proof to prove that $\Theta$ is generated correctly.

(4) To efficiently achieve double-spending tracing without TTP, C constructs a special knowledge proof of $e_{1}$, i.e., $P K_{\Theta}\left(e_{1}\right)$, which is related to $\Theta$. The special property of $P K_{\Theta}\left(e_{1}\right)$ is that if showing $P K_{\Theta}\left(e_{1}\right)$ with the same $\Theta$ twice, i.e., spending the same coin twice, the knowledge of $\left(e_{1}, e_{2}\right)$ is leaked. The details of $P K_{\Theta}\left(e_{1}\right)$ are provided in the following part. 


\section{$\diamond \underline{\text { Deposit Protocol }}$}

$\mathrm{S}$ sends $\mathrm{B}$ the information generated by $\mathrm{C}$ in the above payment protocol. B verifies it as $\mathrm{S}$ does in payment protocol and makes sure that the coin is spent only once.

\section{$\diamond$ Loss Register Protocol}

For registering to trace his lost coins, $\mathrm{C}$ sends $\mathrm{B}$ the information of remaining coins, i.e., $L R_{x}$, which is just shown in loss register protocol so that it does not affect C's anonymity when spending the remaining coins. Then $\mathrm{B}$ sends $\mathrm{C}$ the $\operatorname{ElGamal}_{\text {РК }}\left(g^{e_{2}}\right)$ and the related zero-knowledge proof of it. After verifying the related zero-knowledge proof, $\mathrm{C}$ believes that the $\operatorname{ElGamal}_{\mathrm{PKC}_{\mathrm{C}}}\left(g^{e_{2}}\right)$ was generated by himself in withdrawal protocol and then uses his private key to decrypt $\operatorname{ElGamal}_{\mathrm{PK}}\left(g^{e_{2}}\right)$ so as to get the $g^{e_{2}}$.

\section{$\diamond$ Practical Complete Tracing}

- Unconditional tracing

Unconditional coin-tracing: Getting the information of withdrawal from bank, T uses the private key to decrypt ElGamal $\mathrm{PK}_{\mathrm{T}}\left(g^{e_{2}}\right)$ and publishes $g^{e_{2}}$. In payment protocol, $T_{2}=$ $g^{H(J \| r) e_{2}}\left(\bmod n_{\mathrm{T}}\right)$ will be provided to S with the shown $J$ and $r$, so $T_{2}$ can be identified if $g^{e_{2}}$ is known, that is to say, all the coins can be traced.

Unconditional owner-tracing: Getting the information of deposit from shop, $\mathrm{T}$ uses the factor knowledge of $n_{\mathrm{T}}$ to compute the inverse of $H(J \| r)$ so as to get $g^{e_{2}}$ from $T_{2}$, then T can identify the owner according to withdrawal database.

- Double-spending tracing

Double-spender-tracing: If $\mathrm{C}$ double-spends a coin, he has to use the same $J$ more than once, that is, he has to show the same $\Theta=\operatorname{PRF}\left(e_{2}, J\right)$ more than once, so it can be found out by B. As mentioned earlier, if showing the special knowledge proof $P K_{\Theta}\left(e_{1}\right)$ with the same $\Theta$ twice, $\left(e_{1}, e_{2}\right)$ will be leaked. Since $e_{1}$ is used as tracing information of customer in withdrawal protocol, the double-spender can be traced.

Double-spender's coin-tracing: With the leaked $e_{2}$, every $\Theta=\operatorname{PRF}\left(e_{2}, J\right)$ is computed and published for $J \in\left[0,2^{l}-1\right]$ so that the coins from double-spender cannot be spent anymore.

- Lost-coin tracing

After loss register, B publishes $g^{e_{2}}$. In payment protocol, $T_{2}=g^{H(J \| r) e_{2}}\left(\bmod n_{\mathrm{T}}\right)$ is provided to $\mathrm{S}$ with the shown $J$ and $r$, S can identify $T_{2}$ after $g^{e_{2}}$ is published so that the lost coins cannot be spent by others.

Then, we provide the detailed scheme.

\subsection{System Setup}

Let $\varepsilon>1, k, l_{s}, l_{p}$ and $l_{p s}$ be security parameters. $\lambda_{1}, \lambda_{2}, \gamma_{1}$ and $\gamma_{2}$ denote bit-length satisfying $\lambda_{1}>\varepsilon\left(\lambda_{2}+k\right)+2, \gamma_{1}=l_{p s}+2, \gamma_{1}>\varepsilon\left(\gamma_{2}+k\right)+2, \gamma_{2}, \lambda_{2}>k$. And $\Lambda=\left[2^{\lambda_{1}}-2^{\lambda_{2}}, 2^{\lambda_{1}}+2^{\lambda_{2}}\right]$ and $\Gamma=\left[2^{\gamma_{1}}-2^{\gamma_{2}}, 2^{\gamma_{1}}+2^{\gamma_{2}}\right]$. $H\left(\right.$ ) is a hash function: $\{0,1\}^{*} \rightarrow\{0,1\}^{k}$.

\section{B (bank)'s Setup:}

Select random secret $l_{p}$-bits primes $p^{\prime}$ and $q^{\prime}$, and $p=2 p^{\prime}+1, q=2 q^{\prime}+1$ are primes. Provide the zero-knowledge proof to prove that $n=p q$ is the product of two safe primes [39].

\section{T (trusted authority)'s Setup:}

Select a random $l_{p s}$-bits prime $n_{s}$, and $l_{p s}=\varepsilon\left(\lambda_{2}+k\right)+2 k+2>\lambda_{1}$. Select two secret $l_{p}$-bits primes $p^{\prime \prime}$ and $q^{\prime \prime}$ such that $p_{\mathrm{T}}=2 p^{\prime \prime}+1$ and $q_{\mathrm{T}}=2 q^{\prime \prime}+1$ are primes. Prove that $n_{\mathrm{T}}=p_{\mathrm{T}} q_{\mathrm{T}}$ is the product of two safe primes [39] with the zero-knowledge proof. Choose the elements $a, a_{0}, a_{1}, a_{2}$ of $Q R(n)$ of order $p^{\prime} q^{\prime}, g$ of $Q R\left(n_{\mathrm{T}}\right)$ of order $p^{\prime \prime} q^{\prime \prime}[35] . K_{1} \in_{R} I_{l_{s}}$ and set $h=g^{K_{1}}\left(\bmod n_{\mathrm{T}}\right)$. 


\section{C (customer)'s Setup:}

$S K_{\mathrm{C}} \in{ }_{R} I_{l_{s}}$ and set $P K_{\mathrm{C}}=g^{S K_{\mathrm{C}}}\left(\bmod n_{\mathrm{T}}\right)$.

The system public parameters are $P K=\left(n, n_{s}, n_{\mathrm{T}}, a, a_{0}, a_{1}, a_{2}, g, h\right)$, B's private key is $p^{\prime}$, T's private key is $\left(p^{\prime \prime}, K_{1}\right)$, and C's private key is $S K_{\mathrm{C}}$.

\subsection{Withdrawal Protocol}

As Fig. 3 presents, $\mathrm{C}$ and $\mathrm{B}$ generate cash parameters $\left(x, e_{1}, e_{2}\right)$ together, then $\mathrm{B}$ computes the CL signature [38] (i.e., $(A, e))$. As mentioned in section 6.1, $\left(A_{1}, A_{2}\right)$ is $\operatorname{ElGamal}_{\mathrm{PK}_{C}}\left(g^{e_{2}}\right)$ which encrypts $g^{e_{2}}$ using C's public key $P K_{\mathrm{C}}$ for loss tracing and $\left(A_{2}, A_{3}\right)$ is $\operatorname{ElGamal}_{\mathrm{PK}_{\mathrm{T}}}\left(g^{e_{2}}\right)$ which encrypts $g^{e_{2}}$ using T's public key $h$ for unconditional tracing. Then C gets $\left(x, e_{1}, e_{2}, A, e, r_{\mathrm{C}}\right)$ satisfying $A^{e} \equiv a_{0} a^{x} a_{1}^{e_{1}} a_{2}^{e_{2}}(\bmod n)$, and $r_{\mathrm{C}}$ is the series number of wallet. $W$ and $W_{1}$ guarantee the correctness of computations, and $W$ uses the method in [35] to prove the value range of $e_{1}$.

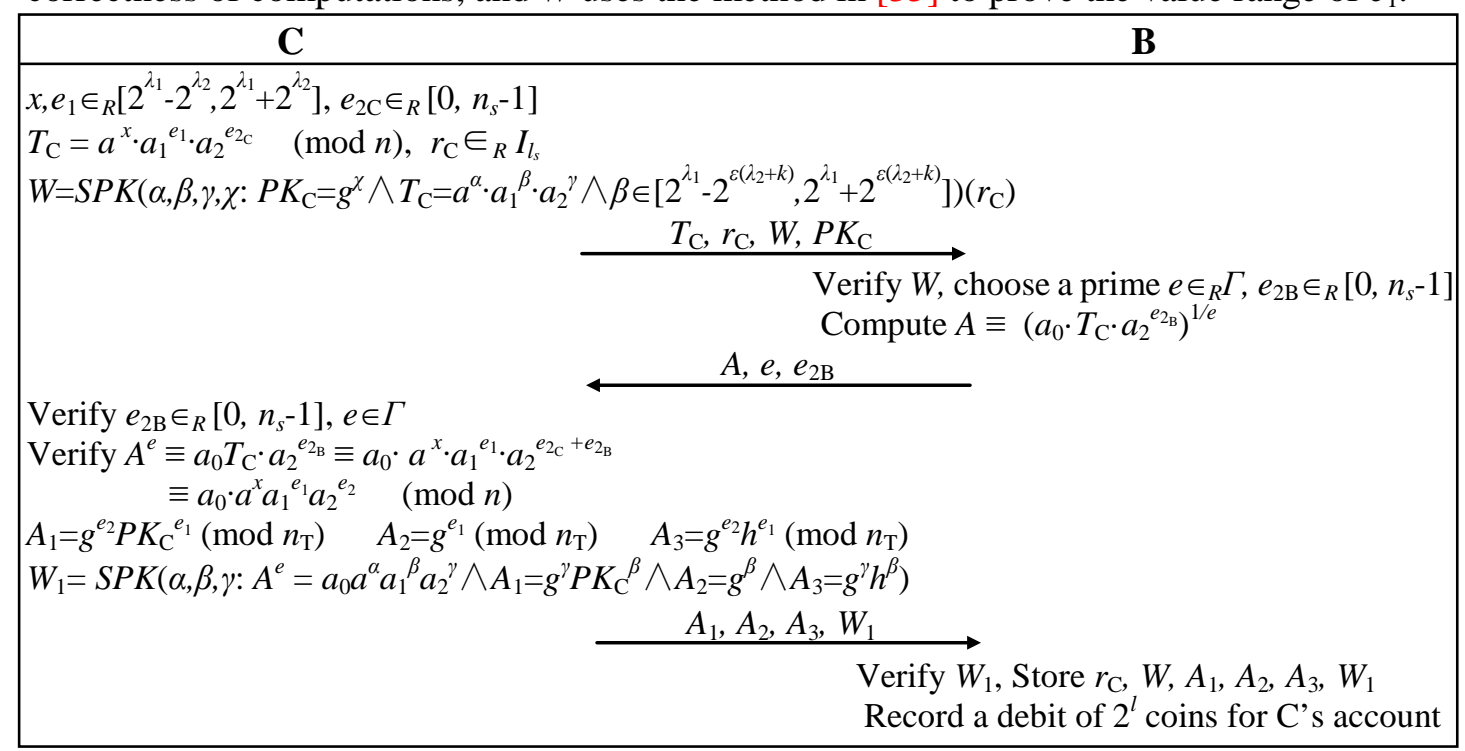

Fig. 3. Withdrawal protocol ( $\in_{R}$ denotes choosing at random)

\subsection{Payment Protocol}

As we sketched in the section 6.1,

(1) To prove the validity of coin anonymously, C conceals the wallet $T_{1}=A h^{w}(\bmod n)$ [8] and provides the zero-knowledge proof of it- since $A^{e} \equiv a_{0} a^{x} a_{1}{ }^{e_{1}} a_{2}{ }^{e_{2}}(\bmod n)$, C provides $P K_{1}\left(e, x, e_{1}, e_{2}, e w: a_{0}=T_{1}^{e} /\left(a^{x} a_{1}^{e_{1}} a_{2}^{e_{2}} h^{e w}\right)\right)$ as the zero-knowledge proof of the wallet ownership. For simplifying denotation, we directly use the parameter name in $P K($ ).

(2) To achieve loss tracing and unconditional tracing, C computes $T_{2}=g^{H(J \| r) e_{2}}\left(\bmod n_{\mathrm{T}}\right)$ with the random $r$. C also provides the zero-knowledge proof $P K_{2}\left(e_{2}: T_{2}=g^{H(J \| r) e_{2}}\right)$.

(3) To prevent double-spending, C provides the serial number of coin $\Theta=a_{1 J}\left(J+e_{2}\right)^{-1}\left(\bmod n_{s}\right)$ $(\bmod n)$ with $J \in\left[0,2^{l}-1\right]$ and $P K_{3}\left(e_{2}: \Theta=a_{1 J}^{\left(J+e_{2}\right)^{-1}\left(\bmod n_{s}\right)}\right)$ proving the correctness of $\Theta$.

Since $\Theta^{\left(J+e_{2}\right)}=a_{1 J}\left(J+e_{2}\right)^{-1}\left(\bmod n_{s}\right)\left(J+e_{2}\right)=a_{1 J}^{t \cdot n_{s}+1}(\bmod n) \quad t \in \mathrm{Z}$

$$
\Theta^{e_{2}}=a_{1 J}^{t \cdot n_{s}+1} / \Theta^{J}(\bmod n)
$$

That is to say, $P K_{3}\left(e_{2}: \Theta=a_{1 J}^{\left(J+e_{2}\right)^{-1}\left(\bmod n_{s}\right)}\right)=P K\left(t, e_{2}: T=a_{1 J}{ }^{t \cdot n_{s}+1} \wedge \Theta^{e_{2}}=T / \Theta^{J}\right)$.

(4) To achieve double-spending-tracing without TTP, $C$ constructs a special knowledge proof $P K_{\Theta}\left(e_{1}\right)$. It leaks $\left(e_{1}, e_{2}\right)$ if showing $P K_{\Theta}\left(e_{1}\right)$ with the same $\Theta$ twice. Now we clarify it. 
According to Definition 3, to prove the discrete logarithm knowledge in $y=g^{\alpha}$, first, prover shows verifier the commitment $g^{r}$ with a random integer $r$, after receiving the random tr from verifier, gets the challenge $c=H\left(y\|g\| g^{r} \| t r\right)$ and then computes the challenge-response $s=r$-cx. Therefore, $g^{r}=g^{s} y^{c}$ (or $c=H\left(y\|g\| g^{s} y^{c} \| t r\right)$ ) is the knowledge proof of $y=g^{\alpha}$.

The idea of constructing $P K_{\Theta}\left(e_{1}\right)$ is to replace $r$ with $\left(J+e_{2}\right)^{-1}$ (mod $\left.n_{s}\right)$ in the knowledge proof of $e_{1}$. If showing $P K_{\Theta}\left(e_{1}\right)$ with the same $\Theta$ twice, there are two challenge-response equations: $s_{\sigma}=\left(J+e_{2}\right)^{-1}\left(\bmod n_{s}\right)-c\left(e_{1}-2^{\lambda_{1}}\right)$ and $s_{\sigma}^{\prime}=\left(J+e_{2}\right)^{-1}\left(\bmod n_{s}\right)-c^{\prime}\left(e_{1}-2^{\lambda_{1}}\right)$, so it is easy to compute $\left(e_{1}, e_{2}\right)$ from the two challenge-response equations. For achieving it, $\mathrm{C}$ computes $d_{1}=T_{1}^{r_{1}} /\left[a^{r_{2}} a_{1}{ }^{\left(J+e_{2}\right)^{-1}\left(\bmod n_{s}\right)} a_{2}{ }^{r_{3}} h^{r_{4}}\right]$ as the commitment of $P K_{1}\left(e, x, e_{1}, e_{2}, e w: a_{0}=T_{1}^{e} /\left(a^{x} a_{1}{ }^{e_{1}} a_{2}{ }^{e_{2}} h^{e w}\right)\right)$, that is to say, $\left(J+e_{2}\right)^{-1}\left(\bmod n_{s}\right)$ replaces $r$ in $d_{1}$. And C must provide the zero-knowledge proof $P K_{4}\left(\delta: \Theta=a_{1 J}{ }^{\delta} \wedge d_{1}=T_{1}{ }_{1} 1\left(a^{r_{2}} a_{1}{ }^{\delta} a_{2}{ }^{r_{3}} h^{r_{4}}\right)\right)$. So $P K_{3}$ and $P K_{4}$ guarantee that one challenge-response of $P K_{1}$ is $s_{\sigma}=\left(J+e_{2}\right)^{-1}\left(\bmod n_{s}\right)-c\left(e_{1}-2^{\lambda_{1}}\right)$.

The special design changes the construction of zero-knowledge proof. So we must prove:

- the security of construction of zero-knowledge proof is preserved;

- $\quad\left(J+e_{2}\right)^{-1}\left(\bmod n_{s}\right)$ can be used as a pseudorandom function in this construction;

- the special knowledge proof has the zero-knowledge property.

The proofs of the above security properties are provided in the section 7 .

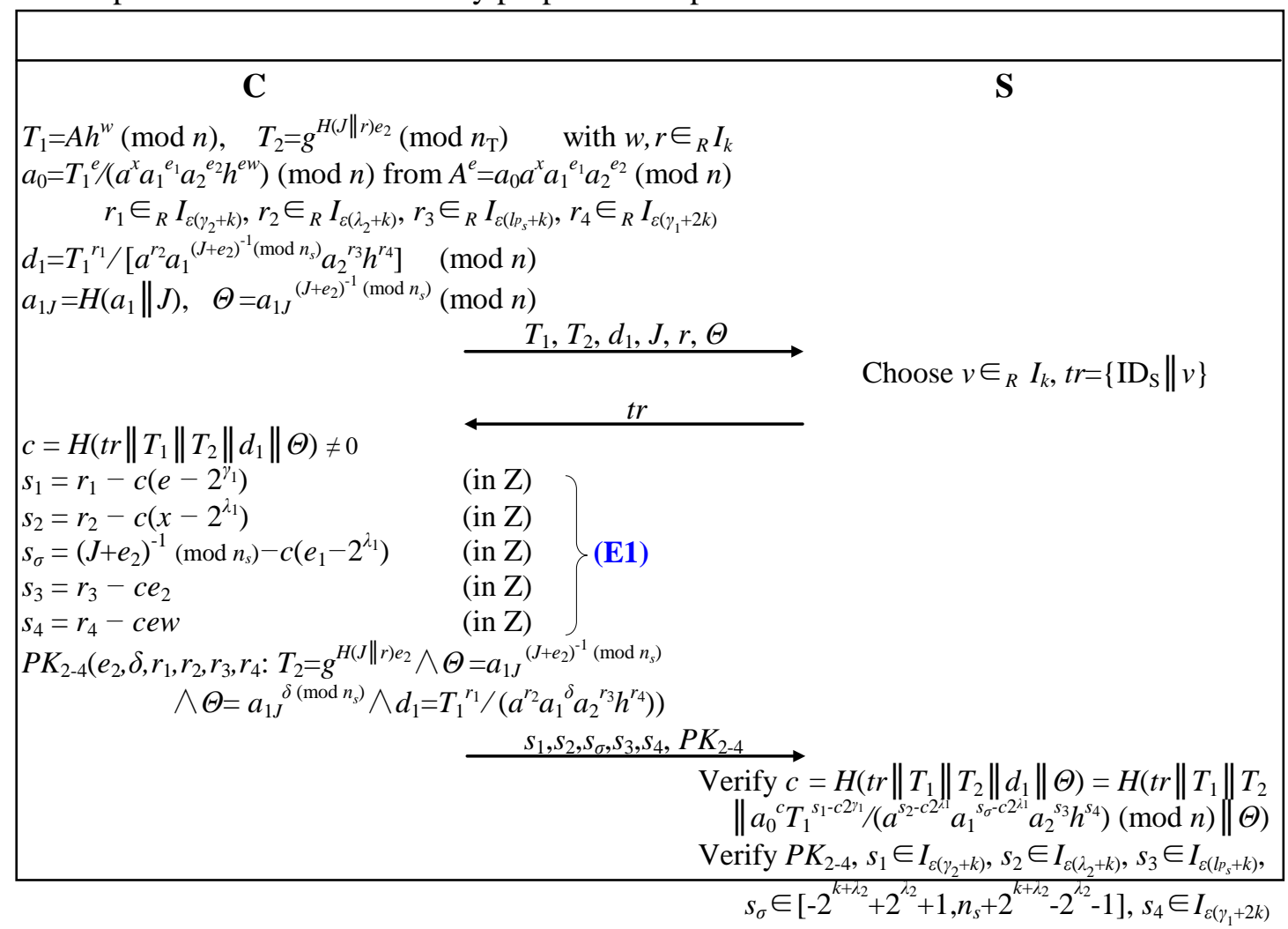

Fig. 4. Payment protocol ( $I_{d}$ denotes $\{0,1\}^{d}$ )

For presenting our idea clearly, only the commitment and challenge-response of $P K_{1}($ ) are provided, since $P K_{\Theta}\left(e_{1}\right)$ is embedded in it. And $P K_{2}() \sim P K_{4}()$ are common zero-knowledge proofs, so the commitment and challenge-response of them are omitted in Fig. 4 (we just use $P K_{2-4}$ to denote the process). In fact, all the commitments are provided in the commitment stage and all the challenge-responses are computed in the challenge-response stage. 


\subsection{Loss Register Protocol}

When $\mathrm{C}$ loses the E-wallet with the series number $r_{\mathrm{C} x}$, he sends $\left(r_{\mathrm{C} 1}, \ldots, r_{\mathrm{C} X-1}, r_{\mathrm{C} X+1}, \ldots, r_{\mathrm{Cn}}\right)$ of his remaining E-wallet to $\mathrm{B}$, and it is shown in Fig. 5. As $r_{\mathrm{C} i}$ is not shown when $\mathrm{C}$ spends E-cash, the anonymity of $\mathrm{C}$ and the unlinkablity of spending will not be influenced. Note that only after B confirms the quantity of all unspent coins in system, that is to say, after a period, C gets the refund from B. In addition, B cannot refuse to perform loss-tracing unless B can provide the payment proof generated before B publishes loss-tracing information in system.

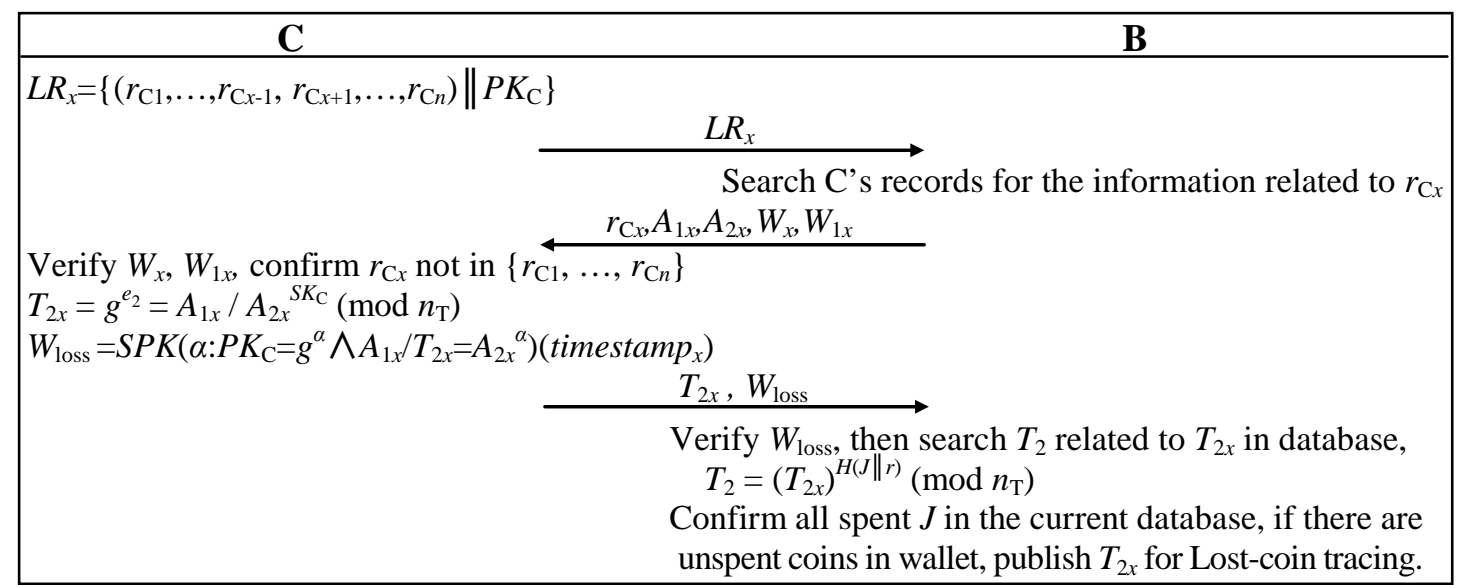

Fig. 5. Loss Register Protocol

\subsection{Deposit Protocol}

$\mathrm{S}$ sends B the payment proof (i.e., Proof $\left.f_{\text {payment }}=\left(T_{1}, T_{2}, J, r, \Theta, t r, c, S_{1}, S_{2}, S_{\sigma}, S_{3}, S_{4}, P K_{2-4}\right)\right)$. B verifies Proof payment as S does in Fig. 4, then B searches $\Theta$ in database. If there is the same $\Theta$ with the same $J$ and a different $c$, it indicates that the Proof $f_{\text {payment }}$ is generated by some double-spender. Therefore, it results in Double-spending tracing. However, if there is the same $(\Theta, J, c)$ in B's database, it indicates that $\mathrm{S}$ deposits this coin twice, and B will abort it. Otherwise, B records the Proof $f_{\text {payment }}$ and terminates this protocol.

\subsection{Complete Tracing}

\section{Double-spending tracing}

If $\mathrm{C}$ spends the same coin twice, $\mathrm{B}$ can trace the double-spender and his coins.

$\diamond \quad$ Double-spender tracing from Bank

$$
\left\{\begin{array}{l}
s_{\sigma}=\left(J+e_{2}\right)^{-1}\left(\bmod n_{s}\right)-c\left(e_{1}-2^{\lambda_{1}}\right) \\
s_{\sigma}^{\prime}=\left(J+e_{2}\right)^{-1}\left(\bmod n_{s}\right)-c^{\prime}\left(e_{1}-2^{\lambda_{1}}\right)
\end{array}\right.
$$

$\mathrm{B}$ can compute $e_{1}$ and $e_{2}$ from the equation set.

$$
\left\{\begin{array}{l}
e_{1}=\left(s_{\sigma}-s_{\sigma}^{\prime}\right) /\left(c^{\prime}-c\right)+2^{\lambda_{1}} \\
e_{2}=\left[s_{\sigma}+c\left(s_{\sigma}-s_{\sigma}^{\prime}\right) /\left(c^{\prime}-c\right)\right]^{-1}\left(\bmod n_{s}\right)-J
\end{array}\right.
$$

Since $A_{2}=g^{e_{1}}\left(\bmod n_{\mathrm{T}}\right), A_{2}=g^{\left(s_{\sigma}-s_{\sigma}^{\prime}\right) /\left(c^{\prime}-c\right)+2 \lambda_{1}}\left(\bmod n_{\mathrm{T}}\right)$.

Therefore, the double-spender with $P K_{\mathrm{C}}$ will be found according to $A_{2}$ stored in withdrawal database. $W_{\text {double }}=\left(\Theta, J, t r, t^{\prime}\right)$ proves that some C double-spends, and $\left(W_{\text {double }},(\mathbf{e} 1), W, W_{1}\right)$ prove that $\mathrm{C}$ with $P K_{\mathrm{C}}$ double-spends.

$\diamond \quad$ Double-spender's coin tracing from Bank

Since $\Theta=a_{1 J}\left(J+e_{2}\right)^{-1}\left(\bmod n_{s}\right)(\bmod n), \Theta^{*}=a_{1 J}\left\{J^{*+}\left[s_{\sigma}+c\left(s_{\sigma}-s_{\sigma}^{\prime}\right) /\left(c^{\prime}-c\right)\right]^{-1}\left(\bmod n_{s}\right)^{-J\}^{-1}}\left(\bmod n_{s}\right)(\bmod n) \ldots(\mathbf{e} 2)\right.$ So B can compute $\Theta^{*}$ for every $J^{*}$ and identify all e-coins in the double-spender's e-wallet. 
And ( $\left.W_{\text {double }},(\mathbf{e} 2)\right)$ prove that the coins belong to a double-spender.

\section{Unconditional tracing}

When E-cash is connected with crimes, $\mathrm{T}$ can do the following tracing.

$\diamond \quad$ Unconditional owner-tracing from $\mathrm{T}$

T gets $J, r$ and $T_{2}$ from payment, and $T_{2}=g^{H(J \| r) e_{2}}\left(\bmod n_{\mathrm{T}}\right)$. Then T uses the private key $p^{\prime \prime}$ to compute $H(J \| r)^{-1}\left(\bmod p^{\prime \prime} q^{\prime \prime}\right), T_{2}^{H(J \| r)^{-1}}=g^{H(J \| r) e_{2} H(J \| r)^{-1}}=g^{e_{2}}=\Omega\left(\bmod n_{\mathrm{T}}\right)$

Searching for $\left(A_{2}, A_{3}\right)$ in withdrawal database, if $A_{3} / A_{2}{ }^{K_{1}}=\Omega\left(\bmod n_{\mathrm{T}}\right)$, T finds the coin owner. $W_{2}=\left(J, r, T_{2},(\mathbf{e} 3)\right)$, and $\left(W, W_{1}, W_{2}\right)$ prove that $C$ with $P K_{\mathrm{C}}$ is the coin owner.

\section{$\diamond \quad$ Unconditional coin-tracing from $\mathrm{T}$}

T gets $A_{2}$ and $A_{3}$ from withdrawal. Since $A_{2}=g^{e_{1}}\left(\bmod n_{\mathrm{T}}\right), A_{3}=g^{e_{2}} h^{e_{1}}\left(\bmod n_{\mathrm{T}}\right), g^{e_{2}}=A_{3} / A_{2}{ }^{K_{1}}$ $\left(\bmod n_{\mathrm{T}}\right)$. Then in payment protocol, $T_{2}=g^{H(J \| r) e_{2}}=\left(A_{3} / A_{2} K_{1}\right)^{H(J \| r)}\left(\bmod n_{\mathrm{T}}\right)$.

So the coin from the wallet will be identified when it is spent. $W_{3}=\operatorname{SPK}\left(\alpha: g^{e_{2}}=A_{3} / A_{2}{ }^{\alpha} \wedge\right.$ $\left.h=g^{\alpha} \wedge T_{2}=\left(A_{3} / A_{2}{ }^{\alpha}\right)^{H(J \| r)}\right)$, and $\left(W_{1}, T_{2}, W_{3}\right)$ prove that the e-coins are from the traced wallet.

\section{Lost-coin tracing}

$\diamond \quad$ Lost-coin tracing from $B$

After Loss Register, all coins in the lost e-wallet will be traced if $T_{2 x}=g^{e_{2}}\left(\bmod n_{\mathrm{T}}\right)$ is published in system. That is to say, $T_{2}=g^{H(J \| r) e_{2}}=\left(T_{2 x}\right)^{H\left(J \|_{r}\right)}\left(\bmod n_{\mathrm{T}}\right)$ in payment.

So the coin from the lost wallet will be identified when it is spent. And $\left(W_{\text {loss }}, W, W_{1}\right)$ prove that the spending coin has been registered for lost-coin tracing by the actual owner with $P K_{\mathrm{C}}$.

\section{Security of the Proposed Scheme}

As we analyzed in section 6.4, the main idea of the proposed scheme is to construct the special knowledge proof. To achieve it, replacing random $r$ with $\left(J+e_{2}\right)^{-1}\left(\bmod n_{s}\right)$ to construct the special challenge-response $s_{\sigma}=\left(J+e_{2}\right)^{-1}\left(\bmod n_{s}\right)-c\left(e_{1}-2^{\lambda_{1}}\right)$. Therefore, the key issues of security are:

- the security of construction of zero-knowledge proof is preserved; (Theorem 1)

- $\quad\left(J+e_{2}\right)^{-1}\left(\bmod n_{s}\right)$ can be used as a pseudorandom function in this construction; (Theorem 2)

- the special knowledge proof has the zero-knowledge property. (Theorem 3)

Then we provide the proofs of the key issues first.

Theorem 1. When each serial number of coin $\Theta$ is used only once, none of proven parameters can be computed from challenge-response equations under discrete logarithm assumption.

Proof. In the payment protocol, $P K_{3}$ proves that $\Theta$ is generated correctly, and $P K_{3}$ and $P K_{4}$ guarantee that one challenge-response of $P K_{1}$ is $S_{\sigma}=\left(J+e_{2}\right)^{-1}\left(\bmod n_{s}\right)-c\left(e_{1}-2^{\lambda_{1}}\right) \ldots \ldots$.(e4). So using a $\Theta$ only once indicate that there is only one equation set (E1) for each $\Theta$, (e4) is the only one that is not a standard challenge-response. Because the other knowledge proofs are the standard knowledge proofs, the related proven parameters cannot be computed from challenge-response equation set. In addition, the single (e4) has arbitrary solutions in their value ranges of $e_{1}$ and $e_{2}$. When $K$ coins from the same wallet are spent, (e4) from the respective equation sets (E1) constitute (E2), and the coefficient matrix $M_{1}$ of (E2) is as follows:

$$
\begin{aligned}
& \text { (E2) }\left\{\begin{array}{c}
\left(J_{1}+e_{2}\right)^{-1}\left(\bmod n_{s}\right)-c_{1} e_{1}=s_{\sigma 1}-c_{1} 2^{\lambda_{1}} \\
\left(J_{2}+e_{2}\right)^{-1}\left(\bmod n_{s}\right)-c_{2} e_{1}=s_{\sigma 2}-c_{2} 2^{\lambda_{1}} \\
\vdots \\
\left(J_{K-1}+e_{2}\right)^{-1}\left(\bmod n_{s}\right)-c_{K-1} e_{1}=s_{\sigma(K-1)}-c_{K-1} 2^{\lambda_{1}} \\
\left(J_{K}+e_{2}\right)^{-1}\left(\bmod n_{s}\right)-c_{K} e_{1}=s_{\sigma K}-c_{K} 2^{\lambda_{1}}
\end{array}\right. \\
& \begin{array}{lllll}
\left(J_{1}+e_{2}\right)^{-1} & \left(J_{2}+e_{2}\right)^{-1} \ldots & \left(J_{K-1}+e_{2}\right)^{-1} & \left(J_{K}+e_{2}\right)^{-1} & e_{1}
\end{array}
\end{aligned}
$$




$$
\left(\begin{array}{cccccc}
1 & 0 & \ldots & 0 & 0 & c_{1} \\
0 & 1 & \ldots & 0 & 0 & c_{2} \\
& & \vdots & & & \\
0 & 0 & \ldots & 1 & 0 & c_{K-1} \\
0 & 0 & \ldots & 0 & 1 & c_{K}
\end{array}\right)
$$

The row vectors of $M_{1}$ are:

$$
\begin{array}{lll}
\alpha_{1}=\left(\begin{array}{llllll}
1 & 0 & \ldots & 0 & 0 & c_{1}
\end{array}\right) & \alpha_{K-1}=\left(\begin{array}{llllll}
0 & 0 & \ldots & 1 & 0 & c_{K-1}
\end{array}\right) \\
\alpha_{2}=\left(\begin{array}{llllllll}
0 & 1 & \ldots & 0 & 0 & c_{2}
\end{array}\right) \ldots \ldots \ldots . . & \alpha_{K}=\left(\begin{array}{llllll}
0 & 0 & \ldots & 0 & 1 & c_{K}
\end{array}\right)
\end{array}
$$

If want to compute $\left(J_{1}+e_{2}\right)^{-1}$, it indicates that there is the matrix as below (the constant $\Delta \neq 0$ ) after elementary transformation, but it will be proven to be infeasible.

$$
\left(\begin{array}{cccccc}
\Delta & 0 & \ldots & 0 & 0 & 0 \\
0 & 1 & \ldots & 0 & 0 & c_{2} \\
& & \vdots & & & \\
0 & 0 & \ldots & 1 & 0 & c_{K-1} \\
0 & 0 & \ldots & 0 & 1 & c_{K}
\end{array}\right)
$$

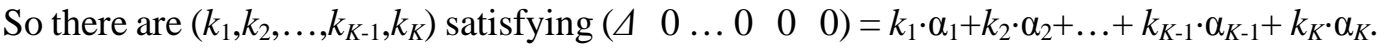

That is to say, $\quad\left(\begin{array}{ccc}k_{1}+0+\ldots+0+0 & =\Delta \\ 0 & +k_{2}+\ldots+0\end{array}\right.$

$$
\left\{\begin{array}{c}
0+k_{2}+\ldots+0+0=0 \\
\vdots \\
0+0+\ldots+k_{K-1}+0=0 \\
0+0+\ldots+0+k_{K}=0 \\
k_{1} C_{1}+k_{2} C_{2}+\ldots+k_{K-1} C_{K-1}+k_{K} C_{K}=0
\end{array}\right.
$$

We have $k_{2}=k_{3}=\ldots=k_{K-1}=k_{K}=0, k_{1}=\Delta \neq 0$, so $c_{1}=0$. However, it will be found by the customer when spending the coin if $c=0$. Therefore, computing $\left(J_{1}+e_{2}\right)^{-1}\left(\bmod n_{s}\right)$ is infeasible. And for the same reason, $\left(J_{i}+e_{2}\right)^{-1}\left(\bmod n_{s}\right)$ can not be figured out, where $2 \leq i \leq K$.

Anyone who tries to compute $e_{1}$ must get the following coefficient matrix $(\Delta \neq 0)$.

$$
\left(\begin{array}{cccccc}
\left(J_{1}+e_{2}\right)^{-1} & \left(J_{2}+e_{2}\right)^{-1} \ldots & \left(J_{K-1}+e_{2}\right)^{-1} & \left(J_{K}+e_{2}\right)^{-1} & e_{1} \\
0 & 0 & \ldots & 0 & 0 & \Delta \\
& 1 & \ldots & 0 & 0 & c_{2} \\
0 & 0 & \vdots & & & \\
0 & 0 & \ldots & 0 & 1 & c_{K-1} \\
0 & & 0 & 1 & c_{K}
\end{array}\right)
$$

So there are $\left(k_{1}, k_{2}, \ldots, k_{K-1}, k_{K}\right)$ satisfying $\left(\begin{array}{llllll}0 & 0 & \ldots & 0 & 0 & \Delta\end{array}\right)=k_{1} \cdot \alpha_{1}+k_{2} \cdot \alpha_{2}+\ldots+k_{K-1} \cdot \alpha_{K-1}+k_{K} \cdot \alpha_{K}$.

That is to say, $\quad\left(\begin{array}{ll}k_{1}+0+\ldots+0+0 & =0\end{array}\right.$

$$
\left\{\begin{array}{c}
0+k_{2}+\ldots+0+0=0 \\
\vdots \\
0+0+\ldots+k_{K-1}+0=0 \\
0+0+\ldots+0+k_{K}=0 \\
k_{1} c_{1}+k_{2} c_{2}+\ldots+k_{K-1} c_{K-1}+k_{K} c_{K}=\Delta
\end{array}\right.
$$

We have $k_{1}=k_{2}=\ldots=k_{K-1}=k_{K}=0$, and it contradicts $\Delta \neq 0$, so figuring out $e_{1}$ is also infeasible.

So $e_{1}$ and $e_{2}$ cannot be computed from (E1) or (E2). Therefore, none of proven parameters can be computed if the customer never spends the same coin twice.

Theorem 2. Suppose ( $\left.s(k), 2^{a(k)}, \varepsilon(k)\right)$-DDHI assumption holds. (i) $f_{1 e_{2}}(J)=a^{\left(J+e_{2}\right)^{-1}}$ is the $\left(s^{\prime}(k)\right.$, $\left.\varepsilon^{\prime}(k)\right)$ pseudo-random function for the one who has no knowledge of $e_{2}$, and $s^{\prime}(k)=$ $s(k) /\left[2^{a(k)} \cdot p o l y(k)\right], \varepsilon^{\prime}(k)=2^{a(k)} \cdot \varepsilon(k)$. (ii) If the one who has no knowledge of $e_{2}$ cannot use the extended Euclidean algorithm to compute inverse of $f_{2 e_{2}}(J), f_{2 e_{2}}(J)=\left(J+e_{2}\right)^{-1}\left(\bmod n_{s}\right)$ is the $\left(s^{\prime \prime}(k)\right.$, $\left.\varepsilon^{\prime \prime}(k)\right)$ pseudorandom function, and $s^{\prime \prime}(k)=s(k) /\left[2^{a(k)} \cdot \operatorname{poly}(k)\right], \varepsilon^{\prime \prime}(k)=2^{a(k)} \cdot \varepsilon(k)$. 
Proof . For the sake of contradiction, suppose there exists the algorithm $\mathcal{A}$, which $\left(s^{\prime}(k), q\right.$, $\left.\varepsilon^{\prime}(k)\right)$-breaks the pseudorandom function, i.e., runs in $s^{\prime}(k)$ time, and can identify $f_{1 e_{2}}\left(J_{0}\right)=$ $a^{\left(J_{0}+e_{2}\right)^{-1}}$ with the probability at least $1 / 2+\varepsilon^{\prime}(k)$, where $\left(J_{0}, a^{\left(J_{0}+e_{2}\right)^{-1}}\right)$ is any unseen point of the function $f_{1 e_{2}}(J)$. Then we can construct the algorithm $\mathcal{B}$, which interacts with $\mathcal{A}$ to break the $q$-DDHI assumption — based on a random instance $\left(a, a^{\alpha}, \ldots, a^{\left(a^{q}\right)}\right)$ of the $q$-DHI problem, the goal of algorithm $\boldsymbol{Z}$ is to identify $a^{1 / \alpha}$. Let $J_{0}=\alpha-e_{2}$ where $e_{2}$ and $\alpha$ are unknown to $\mathcal{A}$ and $\mathcal{Z}$. And the error probability could be decreased by changing $J_{0}$ and executing this algorithm sufficiently many times. Note that $\left(a, a^{e_{2}}, \ldots, a^{\left(e_{2}{ }^{q}\right)}\right)$ can be computed from $\left(a, a^{\alpha}, \ldots, a^{\left(a^{q}\right)}\right)$ according to Binomial Theorem.

Input to the reduction: $\left(a, a^{\alpha}, \ldots, a^{\left(a^{q}\right)}, \Gamma\right) \in G^{q+2}$, where $\Gamma$ is either $a^{1 / \alpha}$ or a random element in $G$. Its goal is to output 1 if $\Gamma=a^{1 / \alpha}$ and 0 otherwise.

Then $\boldsymbol{B}$ interacts with $\mathcal{A}$ as follows:

Query: $\mathcal{A}$ outputs a list of distinct $q_{s}$ serial numbers $J_{1}, \ldots, J_{q s}$, and $q_{s}<q$. Because $\mathscr{A}$ reveals the queries in advance, we could assume $\mathcal{A}$ outputs $q-1$ serial numbers to be responded (if the number is less, reduce the value of $q$ to satisfy $q=q_{s}+1$ ).

Response: $\mathcal{Z}$ computes the polynomial $f(y)=\prod_{i=1}^{q-1}\left(y+J_{i}\right)$.

Then we have $f(y)=\sum_{i=0}^{q-1} \alpha_{i} y^{i}$ by expanding $f(y)$, where $\alpha_{0}, \ldots, \alpha_{q-1} \in Z_{n}$ are the coefficients of the polynomial $f(y)$.

$$
a^{\prime}=\prod_{i=0}^{q-1} a^{\alpha_{i} e_{2}^{i}}=a^{f\left(e_{2}\right)} \quad \prod_{i=1}^{q} a^{\alpha_{i-1} e_{2}^{i}}=a^{e_{2} f\left(e_{2}\right)}=\left(a^{\prime}\right)^{e_{2}}
$$

Then give $a^{\prime}$ to $\mathcal{A}$. Ans we can assume that $f\left(e_{2}\right) \neq 0$, because otherwise, $e_{2}=-J_{i}$ for some $i$ means $\boldsymbol{Z}$ has got the secret $e_{2}$. Then for each $i=1, \ldots, q-1, \boldsymbol{Z}$ computes $R_{i}$ and responds it to $\mathcal{A}$ as follows: let $f_{i}(y)$ be the polynomial $f_{i}(y)=f(y) /\left(y+J_{i}\right)=\prod_{j=1, j \neq i}\left(y^{q-1} J_{i}\right)$. We can expand it as $f_{i}(y)=\sum_{j=0}^{q-2} \beta_{j} y^{j}$.

$$
R_{i}=\prod_{j=0}^{q-2} a^{\beta_{j} e_{2} j}=a^{f_{i}\left(e_{2}\right)}=\left(a^{\prime}\right)^{1 /\left(e_{2}+J_{i}\right)}
$$

So $\mathcal{Z}$ can give $A$ the $q-1$ responses $R_{1}, \ldots, R_{q-1}$ without the knowledge of $e_{2}$.

Challenge: $A$ claims that he can distinguish $a^{1^{1 /\left(e_{2}+J_{*}\right)}}=a^{f\left(e_{2}\right) /\left(e_{2}+J_{*}\right)}$ from a random element in $G$. If $J_{*} \neq J_{0}$, then repeats the algorithm again. Otherwise, $\mathcal{A}$ claims that he can distinguish $a^{1 /\left(e_{2}+J_{0}\right)}=a^{f\left(e_{2}\right) /\left(e_{2}+J_{0}\right)}$ from a random element in $G$ where $J_{0} \notin\left\{J_{1}, \ldots, J_{q-1}\right\}$. Now compute

$$
f(y) /\left(y+J_{0}\right)=\sum_{i=0}^{q-2} \gamma_{i} y^{i}+\gamma_{-1} /\left(y+J_{0}\right)
$$

where $\gamma_{-1} \neq 0$ since $f(y)=\prod_{i=1}^{q-1}\left(y+J_{i}\right)$ and $J_{0} \notin\left\{J_{1}, \ldots, J_{q-1}\right\}$. So

$a^{1 /\left(e_{2}+J_{0}\right)}=a^{f\left(e_{2}\right) /\left(e_{2}+J_{0}\right)}=a^{\sum \begin{array}{ll}q=0 \\ =\end{array}} \gamma_{i} e_{2}^{i+\gamma_{-1}\left(e_{2}+J_{0}\right)}=\Gamma_{0} \cdot a^{\gamma_{-1}\left(e_{2}+J_{0}\right)}=\Gamma_{0} \cdot\left(a^{1 / \alpha}\right)^{\gamma_{-1}}$

Because $\Gamma_{0}=\prod_{i=0}^{q-2}\left(a^{e_{2} i}\right)^{\gamma_{i}}$ is computed from this algorithm, if $\Delta=a^{1 /\left(e_{2}+J_{0}\right)}$ and $\Gamma=a^{1 / \alpha}, \Delta=\Gamma_{0} \cdot \Gamma^{\gamma_{1}}$. So $\mathbb{Z}$ can distinguish $a^{1 / \alpha}$ from $\left(a, a^{\alpha}, \ldots, a^{\alpha q}\right)$ according to the guess about $\Delta^{\stackrel{?}{=}} a^{1 /\left(e_{2}+J_{0}\right)}$ from $\mathcal{A}$.

Guess: $\mathcal{A}$ outputs a guess $b \in\{0,1\}$ for $\Delta$, and then $\boldsymbol{B}$ outputs a guess $b^{\prime} \in\{0,1\}$ for $\Gamma$.

The main running time of the reduction is the time of simulating oracle queries. Since $A$ can make at most $s^{\prime}(k)$ queries, the running time of $\mathcal{Z}$ is $s^{\prime}(k) \cdot\left[2^{a(k)} \cdot p o l y(k)\right]$. And the advantage of $\mathcal{Z}$ is $\varepsilon^{\prime}(k) / 2^{a(k)}$. So $s^{\prime}(k)=s(k) /\left[2^{a(k)}\right.$ poly $\left.(k)\right]$, and $\varepsilon^{\prime}(k)=2^{a(k)} \varepsilon(k)$.

Challenge and Guess following $\mathcal{C}$ : Algorithm $\mathcal{C}$, which $\left(s^{\prime \prime}(k), \varepsilon^{\prime \prime}(k)\right)$-breaks the pseudo-randomness of $f_{2}^{\prime} e_{2}(J)=\left(J+e_{2}\right)^{-1}$, claims to be able to distinguish $P^{*}=\left(J_{0}+e_{2}\right)^{-1}$ from a random element without the knowledge of $e_{2}$ (Theorem 1 guarantees the extended Euclidean algorithm cannot be used). If $\Gamma=a^{1 / \alpha}, a^{P^{* *}}=a^{1 /\left(e_{2}+J_{0}\right)}=\Gamma_{0} \cdot \Gamma^{\gamma_{1}} \ldots \ldots$.(e5). Giving $P^{*}$ to algorithm $\mathcal{C}$, $\mathcal{C}$ outputs a guess $d_{\text {guess }} \in\{0,1\}$ for $P^{*} \stackrel{?}{=}\left(J_{0}+e_{2}\right)^{-1}$. Then $\boldsymbol{Z}$ outputs $d_{\text {guess }}$ for $\Gamma$ according to (e5). 
Therefore, without the knowledge of $e_{2}$, the pseudorandom property of $f_{2}{ }^{\prime} e_{2}(J)=\left(J+e_{2}\right)^{-1}$ is proven if no one can use extended Euclidean algorithm to compute inverse of it, so the pseudorandom property of $f_{2 e_{2}}(J)=\left(J+e_{2}\right)^{-1}\left(\bmod n_{s}\right)$ is proven. Likewise, $f_{2 e_{2}}(J)=\left(J+e_{2}\right)^{-1}\left(\bmod n_{s}\right)$ is a $\left(s^{\prime \prime}(k), \varepsilon^{\prime \prime}(k)\right)$ pseudo-random function, and $s^{\prime \prime}(k)=s(k) /\left[2^{a(k)} \cdot p o l y(k)\right]$ and $\varepsilon^{\prime \prime}(k)=2^{a(k)} \cdot \varepsilon(k)$.

Theorem 3. If customer never spends the same coin twice, the knowledge proof in payment protocol has the statistical zero-knowledge property under q-DDHI assumption.

Proof. We only need to prove that $\left(c, s_{\sigma}\right) \in\{0,1\}^{k} \times\left\{-2^{k+\lambda_{2}}+2^{\lambda_{2}}+1, \ldots, n_{s}+2^{k+\lambda_{2}}-2^{\lambda_{2}}-1\right\}$ from the non-standard knowledge proof has the statistical zero- knowledge property (it will be proven in Theorem 6 that the special knowledge proof is the knowledge proof of $e_{1}$ ). To be concise, suppose $y=a_{1}{ }^{e_{1}},\left(c, s_{\sigma}\right) \in\{0,1\}^{k} \times\left\{-2^{k+\lambda_{2}}+2^{\lambda_{2}}+1, \ldots, n_{s}+2^{k+\lambda_{2}}-2^{\lambda_{2}}-1\right\}$ satisfying $c=H\left(y^{c} \cdot a_{1}{ }^{\sigma_{\sigma}-c 2^{\lambda_{1}}}\right)$.

According to Theorem 2, $\left(J_{i}+e_{2}\right)^{-1}$ in the construction of payment is a random element to the one who has no knowledge of $e_{2}$. The generating mode in the withdrawal protocol guarantees that $\left(J_{i}+e_{2}\right)\left(\bmod n_{s}\right)$ uniformly distributes over [1, $\left.n_{s}-1\right]$, and so does $\left(J_{i}+e_{2}\right)^{-1}\left(\bmod n_{s}\right)$, since every inverse of $\left(J_{i}+e_{2}\right)\left(\bmod n_{s}\right)$ is unique and distinct from each other in [1, $\left.n_{s}-1\right]$.

To prove statistical zero-knowledge property of the knowledge proof, let us show that the simulator which uniformly chooses the challenge, can simulate this protocol-conversation which is statistically indistinguishable from the protocol-conversation with C.

The simulator randomly chooses $\bar{c}$ from $\{0,1\}^{k}$ and $\bar{s}_{\sigma}$ from $\left\{-2^{k+\lambda_{2}}+2^{\lambda_{2}}+1, \ldots, n_{s}+2^{k+\lambda_{2}}-2^{\lambda_{2}}-1\right\}$ satisfying uniform distribution. Using the values, the simulator computes $\bar{\Delta}=y^{\bar{c}} a_{1}{ }^{\bar{s}_{\sigma}-\bar{c}^{\lambda_{1}}}$ (mod $n$ ) [40]. For proving that the values are statistical indistinguishable from a view of a protocol run with C, we will show the probability distribution $P_{S_{\sigma}}\left(s_{\sigma}\right)$ of response $s_{\sigma}$ from C and the probability distribution $P_{\bar{S}_{\sigma}}\left(\bar{s}_{\sigma}\right)$.

In payment protocol, $s_{\sigma}=\left(J+e_{2}\right)^{-1}\left(\bmod n_{s}\right)-c\left(e_{1}-2^{\lambda_{1}}\right)$, where $0<\left(J+e_{2}\right)^{-1}<n_{s}$, and $\left(J+e_{2}\right)^{-1}$ uniformly distributes over $\left[1, n_{s}-1\right]$ as we analyze above. The $e_{1}$ is chosen from $\left[2^{\lambda_{1}}-2^{\lambda_{2}}, 2^{\lambda_{1}}+2^{\lambda_{2}}\right]$, and $c$ (i.e., the output of $H())$ can be any distribution over $\{0,1\}^{k}$. And $l_{p_{s}}=\varepsilon\left(\lambda_{2}+k\right)+2 k+2$.

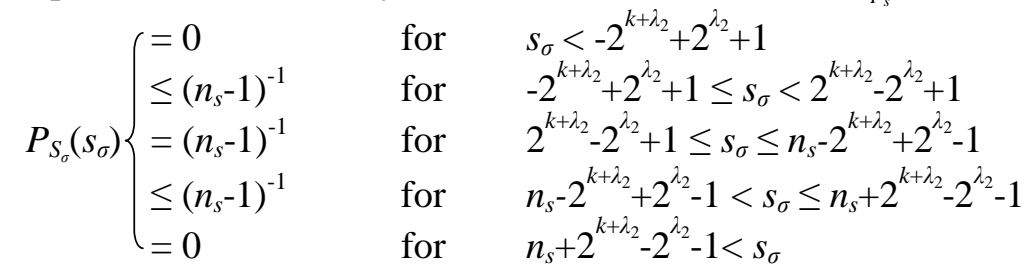

Let us provide a brief explanation of $P_{S_{\sigma}}\left(s_{\sigma}\right)$.

$$
\begin{aligned}
& P\left(s_{\sigma}=2^{k+\lambda_{2}}-2^{\lambda_{2}}+1\right)=\sum P\left(\left(J+e_{2}\right)^{-1}\left(\bmod n_{s}\right)-c\left(e_{1}-2^{\lambda_{1}}\right)=2^{k+\lambda_{2}}-2^{\lambda_{2}}+1\right) \\
= & P\left(\left(J+e_{2}\right)^{-1}\left(\bmod n_{s}\right)=1, c\left(e_{1}-2^{\lambda_{1}}\right)=-2^{k+\lambda_{2}}+2^{\lambda_{2}}\right)+P\left(\left(J+e_{2}\right)^{-1}\left(\bmod n_{s}\right)=2, c\left(e_{1}-2^{\lambda_{1}}\right)=-2^{k+\lambda_{2}}+2^{\lambda_{2}}+1\right) \\
& \quad+\ldots+P\left(\left(J+e_{2}\right)^{-1}\left(\bmod n_{s}\right)=n_{s}-1, c\left(e_{1}-2^{\lambda_{1}}\right)=n_{s}-2^{k+\lambda_{2}}+2^{\lambda_{2}}-2\right) \\
= & \left(n_{s}-1\right)^{-1}\left[P\left(c\left(e_{1}-2^{\lambda_{1}}\right)=-2^{k+\lambda_{2}}+2^{\lambda_{2}}\right)+P\left(c\left(e_{1}-2^{\lambda_{1}}\right)=-2^{k+\lambda_{2}}+2^{\lambda_{2}}+1\right)+\ldots+P\left(c\left(e_{1}-2^{\lambda_{1}}\right)=n_{s}-2^{k+\lambda_{2}}+2^{\lambda_{2}}-2\right)\right] \\
= & \left(n_{s}-1\right)^{-1}\left[P\left(c\left(e_{1}-2^{\lambda_{1}}\right)=-2^{k+\lambda_{2}}+2^{\lambda_{2}}\right)+P\left(c\left(e_{1}-2^{\lambda_{1}}\right)=-2^{k+\lambda_{2}}+2^{\lambda_{2}}+1\right)+\ldots+P\left(c\left(e_{1}-2^{\lambda_{1}}\right)=2^{k+\lambda_{2}}-2^{\lambda_{2}}\right)\right] \\
= & \left(n_{s}-1\right)^{-1} \cdot 1=\left(n_{s}-1\right)^{-1}
\end{aligned}
$$

The Fig. 6 presents the distribution of $P_{\bar{S}_{\sigma}}\left(\bar{s}_{\sigma}\right)$ and $P_{S_{\sigma}}\left(s_{\sigma}\right)$. 


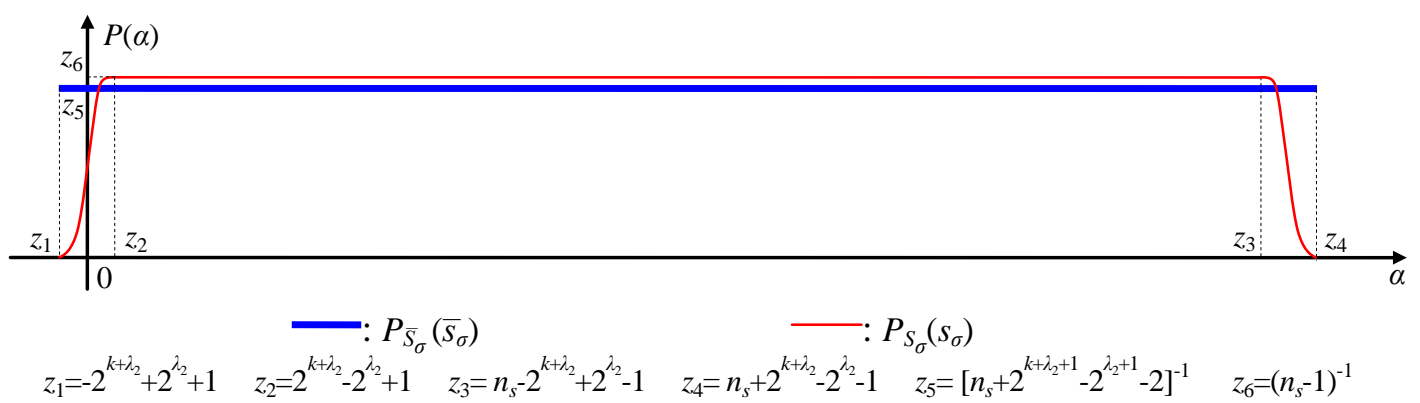

Fig. 6. The Distribution of $P_{\bar{S} \sigma}\left(\bar{s}_{\sigma}\right)$ and $P_{S \sigma}\left(S_{\sigma}\right)$

Then we have

$$
\begin{aligned}
& \sum_{\alpha \in Z}\left|P_{S}(\alpha)-P_{S}(\bar{\alpha})\right|=\sum_{\alpha \in\left\{-2^{k+\lambda_{2}}+2^{\lambda_{2}}+1, \ldots, n_{s}+2^{k+\lambda_{2}}-2^{\lambda_{2}}-1\right\}} P_{s}(\alpha]^{k+\lambda_{2}+1} \mid \\
& <\left(Z_{6}-Z_{5}\right)\left(Z_{3}-Z_{2}+1\right)+Z_{6} \cdot\left[\left(z_{u}-Z_{3}\right)+\left(Z_{2}-Z_{1}\right)\right] \\
& =\left\{\left(n_{s}-1\right)^{-1}-\left[n_{s}+2^{k+\lambda_{2}+1}-2^{\lambda_{2}+1}-2\right]^{-1}\right\} \cdot\left(n_{s}-2^{k+\lambda_{2}+1}+2^{\lambda_{2}+1}-2\right)+\left(n_{s}-1\right)^{-1}\left[\left(2^{k+\lambda_{2}+1}-2^{\lambda_{2}+1}\right)+\left(2^{k+\lambda_{2}+1}-2^{\lambda_{2}+1}\right)\right] \\
& <\left(n_{s}-1\right)^{-1} \cdot\left(2^{k+\lambda_{2}+1}-2^{\lambda_{2}+1}-1\right)+\left(n_{s}-1\right)^{-1}\left(2^{k+\lambda_{2}+2}-2^{\lambda_{2}+2}\right) \\
& =\left(n_{s}-1\right)^{-1} \cdot\left(2^{k+\lambda_{2}+1}-2^{\lambda_{2}+1}-1+2^{k+\lambda_{2}+2}-2^{\lambda_{2}+2}\right)<\left(n_{s}-1\right)^{-1} \cdot\left(2^{k+\lambda_{2}+3}-2^{\lambda_{2}+3}\right)<\left(n_{s}-1\right)^{-1} \cdot 2^{k+\lambda_{2}+3}<1 / 2^{(\varepsilon-1)\left(\lambda_{2}+k\right)+2 k-2}
\end{aligned}
$$

For $\varepsilon>1$, the denominator of last term of the above computation is over a polynomial in input length, so the distributions of $s_{\sigma}$ and $\bar{s}_{\sigma}$ are statistical indistinguishable. Therefore, according to the Definition 2, the interactive protocol of payment is the honest-verifier statistical zero-knowledge proof.

Theorem 4. Under Discrete Logarithm assumption, the non-standard challenge-response $S_{\sigma}=\left(J+e_{2}\right)^{-1}\left(\bmod n_{s}\right)-c\left(e_{1}-2^{\lambda_{1}}\right)$ is computed correctly in the challenge-response equation set (E1). Proof. In payment protocol, $P K_{3}\left(e_{2}: \Theta=a_{1 J}{ }^{\left(J+e_{2}\right)^{-1}\left(\bmod n_{s}\right)}\right)$ and $P K_{4}\left(\delta: \Theta=a_{1 J}{ }^{\delta} \wedge d_{1}=T_{1}^{r_{1}} /\left(a^{r_{2}} a_{1}{ }^{\delta} a_{2}{ }^{r_{3}} h^{r_{4}}\right)\right)$ prove that $d_{1}=T_{1}^{r_{1}}\left[a^{r_{2}} a_{1}^{\left(J+e_{2}\right)^{-1}\left(\bmod n_{s}\right)} a_{2}{ }^{r} h^{r_{4}}\right]$, which is used as a commitment of zero-knowledge proof about the discrete logarithm knowledge of $a_{0}=T_{1}{ }^{e} /\left(a^{x} a_{1}{ }^{e_{1}} a_{2}{ }^{e_{2}} h^{e w}\right)$, C shows $s_{\sigma}$ satisfying

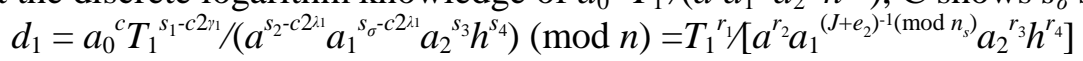

Without the discrete logarithm knowledge of $\left(a_{0}, T_{1}, a, a_{1}, a_{2}, h\right)$ to each other, the exponents of $a_{1}$ are equal: $s_{\sigma}+c\left(e_{1}-2^{\lambda_{1}}\right)=\left(J+e_{2}\right)^{-1}\left(\bmod n_{s}\right)+k_{0} \cdot n_{s} \ldots \ldots .(\mathbf{e 6})$, where $\left(J+e_{2}\right)^{-1}\left(\bmod n_{s}\right)$ denotes the value of the inverse of $\left(J+e_{2}\right)$ is in $\left[1, n_{s}-1\right]$ and $k_{0}$ is any integer. If $k_{0}=0, s_{\sigma}=\left(\underline{J+e_{2}}\right)^{-1}$ (mod $\left.n_{s}\right)-c\left(e_{1}-2^{\lambda_{1}}\right)$ so that the tracing can be performed correctly. We present how to guarantee $k_{0}=0$.

(1) If $\mathrm{C}$ executes payment protocol honestly, $k_{0}=0$. From $c \in\left[1,2^{k}-1\right],\left(e_{1}-2^{\lambda_{1}}\right) \in\left[-2^{\lambda_{2}}, 2^{\lambda_{2}}\right]$, and $\left(J+e_{2}\right)^{-1}\left(\bmod n_{s}\right) \in\left[1, n_{s}-1\right]$, the probability of $s_{\sigma} \in\left[-2^{k+\lambda_{2}}+2^{\lambda_{2}}+1, n_{s}+2^{k+\lambda_{2}}-2^{\lambda_{2}}-1\right]$ is 1 . And $S$ verifies whether $s_{\sigma} \in\left[-2^{k+\lambda_{2}}+2^{\lambda_{2}}+1, n_{s}+2^{k+\lambda_{2}}-2^{\lambda_{2}}-1\right]$ or not in payment protocol.

(2) If $C$ is dishonest and computes (e6) choosing $k_{0} \neq 0$

If $C$ computes (e6) choosing $k_{0}=1$, i.e., $s_{\sigma}=\left(J+e_{2}\right)^{-1}\left(\bmod n_{s}\right)+n_{s}-c\left(e_{1}-2^{\lambda_{1}}\right)$. In this case, if $s_{\sigma} \in\left[-2^{k+\lambda_{2}}+2^{\lambda_{2}}+1, n_{s}+2^{k+\lambda_{2}}-2^{\lambda_{2}}-1\right]$, it means $-n_{s}-2^{k+\lambda_{2}}+2^{\lambda_{2}}+1 \leq\left(\underline{J+e_{2}}\right)^{-1}\left(\bmod n_{s}\right)-c\left(e_{1}-2^{\lambda_{1}}\right) \leq 2^{k+\lambda_{2}}-2^{\lambda_{2}}-1$. We use $\operatorname{Prob}_{k_{0}=1}$ to denote the probability that $S$ cannot find the deceit,

$$
\begin{aligned}
\operatorname{Prob}_{k_{0}=1} & =\operatorname{Prob}\left\{-n_{s}-2^{k+\lambda_{2}}+2^{\lambda_{2}}+1 \leq\left(\underline{J+e_{2}}\right)^{-1}\left(\bmod n_{s}\right)-c\left(e_{1}-2^{\lambda_{1}}\right) \leq 2^{k+\lambda_{2}}-2^{\lambda_{2}}-1\right\} \\
& =\operatorname{Prob}\left\{-2^{k+\lambda_{2}}+2^{\lambda_{2}}+1 \leq\left(\underline{J+e_{2}}\right)^{-1}\left(\bmod n_{s}\right)-c\left(e_{1}-2^{\lambda_{1}}\right) \leq 2^{k+\lambda_{2}}-2^{\lambda_{2}}-1\right\} \\
& =\operatorname{Prob}\left\{1 \leq\left(\underline{J+e_{2}}\right)^{-1}\left(\bmod n_{s}\right) \leq c\left(e_{1}-2^{\lambda_{1}}\right)+2^{k+\lambda_{2}}-2^{\lambda_{2}}-1\right\}
\end{aligned}
$$

$\left.\left(\underline{J}_{2}\right)_{2}\right)^{-1}\left(\bmod n_{s}\right)$ uniformly distributes over $\left[1, n_{s}-1\right]$ since $\left(J+e_{2}\right)\left(\bmod n_{s}\right)$ uniformly distributes over $\left[1, n_{s}-1\right]$ according to the withdrawal protocol, $e_{1} \in\left[2^{\lambda_{1}}-2^{\varepsilon\left(\lambda_{2}+k\right)}, 2^{\lambda_{1}}+2^{\varepsilon\left(\lambda_{2}+k\right)}\right]$ according to $W$. 
Then we have $\left(e_{1}-2^{\lambda_{1}}\right) \in\left[-2^{\varepsilon\left(\lambda_{2}+k\right)}, 2^{\varepsilon\left(\lambda_{2}+k\right)}\right]$. Therefore,

$$
\text { Pro }_{k_{0}=1}<\left\{\left(2^{k}-1\right) \cdot 2^{\varepsilon\left(\lambda_{2}+k\right)}+2^{k+\lambda_{2}}-2^{\lambda_{2}}-1\right\} /\left(n_{s}-1\right)<2^{\varepsilon\left(\lambda_{2}+k\right)+k+1} /\left(n_{s}-1\right)
$$

Because $n_{s}$ is a $l_{p s}$-bits prime, and $l_{p_{s}}=\varepsilon\left(\lambda_{2}+k\right)+2 k+2$,

$\operatorname{PrO}_{k_{0}=1}<2^{\varepsilon\left(\lambda_{2}+k\right)+k+1} /\left(n_{s}-1\right)<1 / 2^{k}$, and it is negligible (usually, bit-length of hash function $k=128$ or 160$)$.

- If $C$ computes (e6) choosing $k_{\underline{0}}=-1$, i.e., $s_{\sigma}=\left(\underline{J+e_{2}}\right)^{-1}\left(\bmod n_{s}\right)-n_{s}-c\left(e_{1}-2^{\lambda_{1}}\right)$. In this case, if $s_{\sigma} \in\left[-2^{k+\lambda_{2}}+2^{\lambda_{2}}+1, n_{s}+2^{k+\lambda_{2}}-2^{\lambda_{2}}-1\right]$, it means $n_{s}-2^{k+\lambda_{2}}+2^{\lambda_{2}}+1 \leq\left(\underline{J+e_{2}}\right)^{-1}\left(\bmod n_{s}\right)-c\left(e_{1}-2^{\lambda_{1}}\right) \leq 2 n_{s}+2^{k+\lambda_{2}}-2^{\lambda_{2}}-1$.

$$
\begin{aligned}
\operatorname{Prob}_{k_{0}=-1} & =\operatorname{Prob}\left\{c\left(e_{1}-2^{\lambda_{1}}\right)+n_{s}-2^{k+\lambda_{2}}+2^{\lambda_{2}}+1 \leq\left(\underline{J+e_{2}}\right)^{-1}\left(\bmod n_{s}\right) \leq n_{s}-1\right\} \\
& <\operatorname{Prob}\left\{\left(2^{k}-1\right) \cdot-2^{\varepsilon\left(\lambda_{2}+k\right)}+n_{s}-2^{k+\lambda_{2}}+2^{\lambda_{2}}+1 \leq\left(J+e_{2}\right)^{-1}\left(\bmod n_{s}\right) \leq n_{s}-1\right\} \\
& <\left[2^{\varepsilon\left(\lambda_{2}+k\right)+k}+2^{k+\lambda_{2}}-2^{\lambda_{2}}-2\right] /\left(n_{s}-1\right)<2^{\varepsilon\left(\lambda_{2}+k\right)+k+1} /\left(n_{s}-1\right)<1 / 2^{k}
\end{aligned}
$$

If $C$ computes (e6) with $\left|k_{0}\right|>1$, it is easy to see that $s_{\sigma} \notin\left[-2^{k+\lambda_{2}}+2^{\lambda_{2}}+1, n_{s}+2^{k+\lambda_{2}}-2^{\lambda_{2}}-1\right]$.

As above, $\operatorname{Prob}_{\left|k_{0}\right|=1}$ is negligible and $\operatorname{Prob}_{\left|\mathrm{k}_{0}\right|>1}=0$. Therefore, $\mathrm{C}$ has to computes (e6) choosing $\underline{k}_{0}=0$, i.e., it guarantees that $s_{\sigma}=\left(\underline{J+e_{2}}\right)^{-1}\left(\bmod n_{s}\right)-c\left(e_{1}-2^{\lambda_{1}}\right)$ is computed correctly.

The proposed scheme is based on our previous work [30]. Considering the similar proofs of Theorem 5-Theorem 8 had been provided in [30], we just present Theorem 5-Theorem 8.

Theorem 5. Under S-RSA assumption, any PPT adversary except $\mathrm{B}$, can not, with nonnegligible probability, computes $\left(e_{1}, e_{2}, x,[A, e], a_{0}\right)$ s.t. $A^{e}=a_{0} a^{x} a_{1}^{e_{1}} a_{2}^{e_{2}}(\bmod n)$ with $e_{1}, x \in \Lambda$, $e_{2} \in\left[0,2 n_{s}-2\right]$ and $e \in \Gamma$ which is different from wallets generated in withdrawal protocol.

Theorem 6. The knowledge proof in the proposed payment protocol is the proof of C's knowledge of his wallet parameters $\left(e_{1}, e_{2}, x,[A, e]\right)$ under $S$-RSA assumption.

Theorem 7. No PPT adversary except $\mathrm{C}$ can, with non-negligible probability, generate the spending proof that is not actually generated by $\mathrm{C}$, but the spending proof is proven to be generated by $\mathrm{C}$ under the Discrete Logarithm assumption.

Theorem 8. Our compact E-cash scheme with (BSetup, TSetup, CSetup, Withdraw, Spend, Deposit, UnconditionallyTrace, LossCoinTrace, DoubleSpendTrace) guarantees Balance, Complete-tracing, Anonymity of customer, Strong Exculpability under S-RSA assumption and $q$-DDHI assumption in random oracle model.

\section{Efficiency Analysis}

\subsection{Storage Space of some E-cash Systems}

To compare clearly, Table 2 presents the storage space of each stage in some E-cash systems. For achieving comparable secure level, the bit-length of order of cyclic group $G$ is 1024 [17, $16,8,20,19,41,26,30]$ and our scheme, and the prime order $p$ of $G_{1}$ and $G_{2}$ in bilinear map is 160 bits in [8, 21, 22, 31, 23]. We select $L=10$ in [22, 26] and select $l=10$ in $[8,21,23]$ and our scheme accordingly, which make these schemes provide the similar functions.

Table 2. Storage space for 1 coin in E-cash schemes

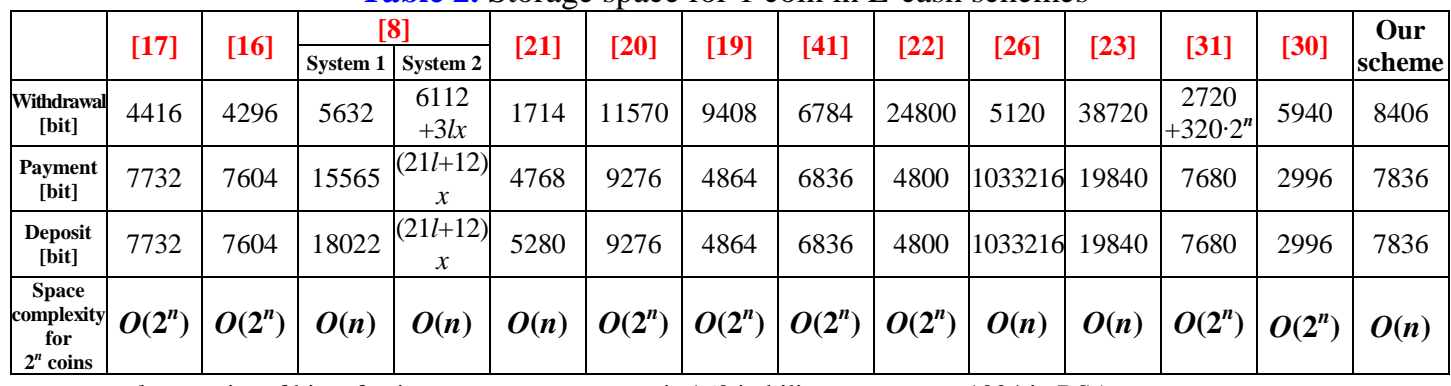

l: quantity of bits of coin counter

$x$ : is 160 in bilinear group, or 1024 in RSA group 
The space complexity for $2^{n}$ coins is $O(n)$ in compact E-cash, that is to say, in [8, 21, 23] and our scheme, the storage space for $2^{n}$ coins is the same as it (shown in Table 2) for 1 coins.

\subsection{Computation Cost of some E-cash Systems}

Since multi-based exponentiations and bilinear pairings are the main computations of the protocols in the systems, they are presented in Table 3, while the slight computations, such as modular addition computations and hash computations, are all neglected.

Table 3. Computation cost for 1 coin in E-cash schemes

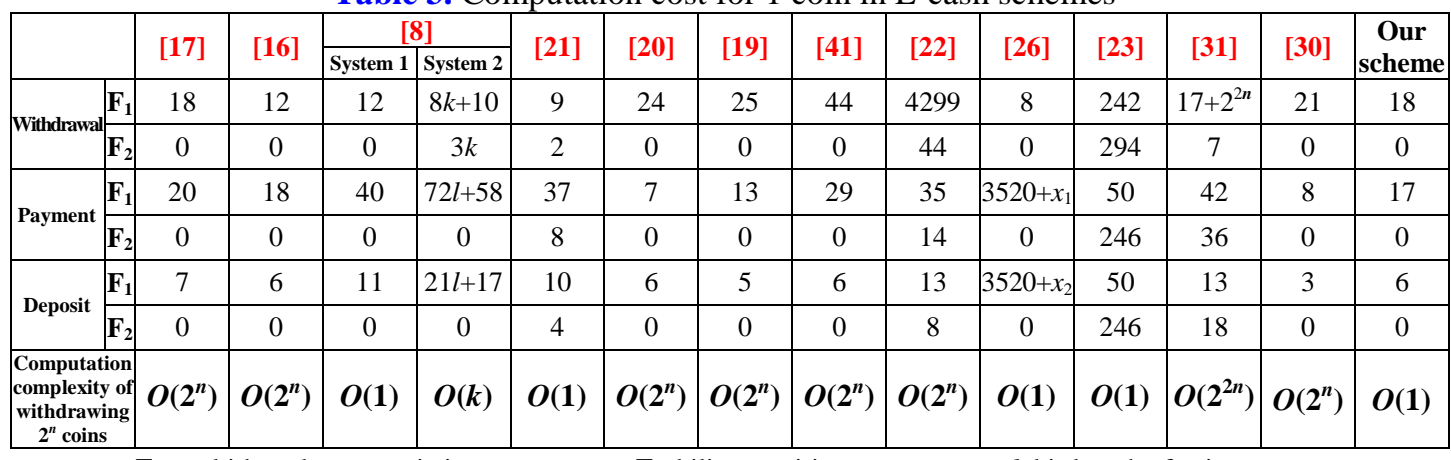

$\mathbf{F}_{1}$ : multi-based exponentiation $\quad \mathbf{F}_{2}$ : bilinear pairing

$k$ : cheating probability is $2^{-k}$ at most in the system2 of [8]

l: bit-length of coin counter

$x_{1}, x_{2}$ : related to system parameters

In compact E-cash system, to withdraw $2^{n}$ coins, the user performs the withdrawal protocol only once, so $[8,21,23]$ and our scheme achieve better efficiency for $2^{n}$ coins. Note that in divisible e-cash, the user can withdraw the coin with the value of $2^{n}$ coins and use $O(n)$ space to store it, but for spending one coin with $1 / 2^{n}$ of total value, the preparation work is costly.

\subsection{Our solution to two problems}

Our main work is to solve two problems, one is achieving the complete and practical tracing, and the other one is solving the efficiency problem caused by tracing customer's coins if he double-spends a coin. Table 4 presents our solution to the practical and complete tracing, that is to say, it presents the available tracing functions in the E-cash schemes.

Table 4. The available tracings in the E-cash systems

\begin{tabular}{|c|c|c|c|c|c|c|c|c|c|c|c|c|c|c|}
\hline & {$[17]$} & {$[16]$} & \multicolumn{2}{|c|}{$[\mathbf{8}]$} & {$[21]$} & {$[20]$} & {$[19]$} & {$[41]$} & {$[22]$} & {$[26]$} & {$[23]$} & {$[31]$} & {$[30]$} & $\begin{array}{c}\text { Our } \\
\text { scheme }\end{array}$ \\
\hline $\begin{array}{c}\text { Double-spender tracing } \\
\text { from Bank }\end{array}$ & N/A & N/A & Yes & Yes & Yes & Yes & Yes & Yes & Yes & Yes & Yes & Yes & Yes & Yes \\
\hline $\begin{array}{c}\text { Double-spender's coin tracing } \\
\text { from Bank }\end{array}$ & N/A & N/A & N/A & Yes & N/A & N/A & N/A & N/A & N/A & N/A & N/A & Yes & N/A & Yes \\
\hline $\begin{array}{c}\text { Unconditional Owner tracing } \\
\text { from TTP }\end{array}$ & Yes & Yes & N/A & N/A & N/A & N/A & N/A & Yes & N/A & N/A & N/A & N/A & Yes & Yes \\
\hline $\begin{array}{c}\text { Unconditional coin tracing } \\
\text { from TTP }\end{array}$ & Yes & N/A & N/A & N/A & N/A & N/A & N/A & Yes & N/A & N/A & N/A & N/A & Yes & Yes \\
\hline $\begin{array}{c}\text { Lost-coin tracing } \\
\text { from Bank }\end{array}$ & N/A & N/A & N/A & N/A & N/A & N/A & N/A & N/A & N/A & N/A & N/A & N/A & Yes & Yes \\
\hline $\begin{array}{c}\text { Compact E-cash which can } \\
\text { trace double-spenders coins }\end{array}$ & & & & $\checkmark$ & & & & & & & & $\checkmark$ & $\checkmark$ \\
\hline
\end{tabular}

According to Table 4, the system 2 of [8], [31] and ours are compact E-cash systems which can trace double-spender's coins (providing it without TTP), and to the best of our knowledge, they include all compact E-cash systems providing this function. Then Table 5 presents the 
solution to the efficiency problem caused by this tracing. And for achieving the comparable secure level, $l=10, k=100, x=1024$ (the meanings of the parameters are in Table 2 and Table 3 ).

Table 5. Compact E-cash with double-spender's coin-tracing

\begin{tabular}{|c|c|c|c|c|c|c|c|c|c|c|}
\hline & & \multirow{2}{*}{\multicolumn{3}{|c|}{ Storage space [bit] }} & \multicolumn{6}{|c|}{ Computation cost } \\
\hline & & & & & \multicolumn{3}{|c|}{$\mathrm{F}_{1}$} & \multicolumn{3}{|c|}{$\mathrm{F}_{2}$} \\
\hline & & $\begin{array}{c}{[8]} \\
\text { System } 2\end{array}$ & [31] & Our scheme & $\begin{array}{c}{[8]} \\
\text { System } 2\end{array}$ & [31] & Our scheme & $\begin{array}{c}{[8]} \\
\text { System } 2\end{array}$ & [31] & Our scheme \\
\hline $\begin{array}{l}\text { For } 2^{n} \\
\text { coins }\end{array}$ & Withdrawal & 36832 & 330400 & 8406 & 810 & 1048593 & 18 & 300 & 7 & 0 \\
\hline \multirow{2}{*}{$\begin{array}{c}\text { For } 1 \\
\text { coin }\end{array}$} & Payment & 227328 & 7680 & 7836 & 778 & 42 & 17 & 0 & 36 & 0 \\
\hline & Deposit & 227328 & 7680 & 7836 & 227 & 13 & 6 & 0 & 18 & 0 \\
\hline
\end{tabular}

\section{Conclusion}

Anonymity is good, but it could be abused for crimes or cause trouble when E-cash is lost. Complete tracing can solve this problem. However, it also threatens the honest user's privacy. The reasonable solution is to separate different tracing functions provided by different entities and choose the available ones according to the circumstances. Practical tracing can achieve it. To achieve the practical and complete tracing, another serious problem must be solved, i.e., how to trace double-spender's coins efficiently. For solving it, we propose the particular knowledge proof, and using it honestly keeps perfect zero-knowledge property, while using it dishonestly leaks the information of proven knowledge. Since it changes the inner construction of standard zero-knowledge proof, we provide the complete proofs of it. Consequently, the practical and complete tracing is also efficient.

\section{References}

[1] Y. Chen, J. S. Chou, H. M. Sun, and M. H. Cho, "A novel electronic cash system with trustee-based anonymity revocation from pairing," Electronic Commerce Research and Applications. vol.10, no.6, pp. 673-682, 2011. Article (CrossRef Link)

[2] Z. Tan, "An Off-line Electronic Cash Scheme Based on Proxy Blind Signature," The Computer Journal, vol. 54, no. 4, pp. 505-512, 2011. Article (CrossRef Link)

[3] D. Chaum, "Blind signatures for untraceable payments," in Proc. of CRYPTO'82, pp. 199-203, 1983. Article (CrossRef Link)

[4] Pin-Chang Su and Chien-Hua Tsai, "New Proxy Blind Signcryption Scheme for Secure Multiple Digital Messages Transmission Based on Elliptic Curve Cryptography," KSII Transactions on Internet and Information Systems, vol. 11, no. 11, pp. 5537-5555, 2017. Article (CrossRef Link)

[5] Md. Abdullah Al Rahat Kutubi, Kazi Md. Rokibul Alam, Rafaf Tahsin, G. G. Md. Nawaz Ali, Peter Han Joo Chong and Yasuhiko Morimoto, "An Offline Electronic Payment System Based on an Untraceable Blind Signature Scheme," KSII Transactions on Internet and Information Systems, vol. 11, no. 5, pp. 2628-2645, 2017. Article (CrossRef Link)

[6] Zhen Zhao, Jie Chen, Yueyu Zhang and Lanjun Dang, "An Efficient Revocable Group Signature Scheme in Vehicular Ad Hoc Networks," KSII Transactions on Internet and Information Systems, vol. 9, no. 10, pp. 4250-4267, 2015. Article (CrossRef Link)

[7] Run Xie, Chunxiang Xu, Chanlian He and Xiaojun Zhang, "An Efficient Dynamic Group Signature with Non-frameability,” KSII Transactions on Internet and Information Systems, vol. 10, no. 5, pp. 2407-2426, 2016. Article (CrossRef Link)

[8] J. Camenisch, S. Hohenberger, and A. Lysyanskaya, "Compact e-cash," in Proc. of Advances in Cryptology-EUROCRYPT 2005, pp. 302-321, 2005. Article (CrossRef Link) 
[9] S. von Solms and D. Naccache, “On blind signatures and perfect crimes," Computers \& Security, vol. 11, pp.581-583, 1992. Article (CrossRef Link)

[10] E. Brickell, P. Gemmell, and D. Kravitz, "Trustee-based tracing extensions to anonymous cash and the making of anonymous change," in Proc. of 6th annual ACM-SIAM symposium on Discrete algorithms, pp. 457-466,1995. Article (CrossRef Link)

[11] M. Stadler, J. Piveteau, and J. Camenisch, "Fair blind signatures," in Proc. of Advances in Cryptology Eurocrypt’95, pp. 209-219, 1995. Article (CrossRef Link)

[12] A. Lysyanskaya and Z. Ramzan, "Group blind digital signatures: A scalable solution to electronic cash,” in Proc. of FC’98, pp. 184-197, 1998. Article (CrossRef Link)

[13] G. Maitland and C. Boyd, "Fair electronic cash based on a group signature scheme,” Information and Communications Security, pp. 461-465, 2001. Article (CrossRef Link)

[14] H. Oros and C. Popescu, "A Secure and Efficient Off-line Electronic Payment System for Wireless Networks,” Intl. J. of Computers, Comm. and Control, Suppl. Issue Vol. V, No. 4, pp. 551-557, 2010. Article (CrossRef Link)

[15] J. Zhang, L. Ma, and Y. Wang, “Fair E-Cash System without Trustees for Multiple Banks,” in Proc. of CISW 2007, pp. 585-587, 2007. Article (CrossRef Link)

[16] S. Canard, C. Delerablée, A. Gouget, E. Hufschmitt, F. Laguillaumie, H. Sibert, J. Traoré, and D. Vergnaud, "Fair E-Cash: Be Compact, Spend Faster," in Proc. of ISC 2009: Information Security, pp. 294-309, 2009. Article (CrossRef Link)

[17] S. Canard and J. Traoré, “On fair e-cash systems based on group signature schemes,” in Proc. of ACISP2003, pp. 237-248, 2003. Article (CrossRef Link)

[18] W. Qiu, K. Chen"A new offline privacy protecting e-cash system with revokable anonymity," Information Security, pp.177, 2002. Article (CrossRef Link)

[19] H. Wang, J. Cao, and Y. Zhang, “A flexible payment scheme and its role-based access control,” IEEE Transactions on Knowledge and Data Engineering, vol. 17, no. 3, pp. 425-436, 2005. Article (CrossRef Link)

[20] J. Liu, P. Tsang, and D. Wong, “Recoverable and untraceable e-cash,” in Proc. of PKI, pp. 206-214, 2005. Article (CrossRef Link)

[21] M. Au, W. Susilo, and Y. Mu, "Practical compact e-cash," in Proc. of the 12th Australasian conference on Information security and privacy 2007, pp. 431-445, 2007. Article (CrossRef Link)

[22] M. Au, W. Susilo, and Y. Mu, "Practical anonymous divisible e-cash from bounded accumulators,” Financial Cryptography and Data Security, pp. 287-301, 2008. Article (CrossRef Link)

[23] M. Belenkiy, M. Chase, M. Kohlweiss, and A. Lysyanskaya, "Compact e-cash and simulatable VRFs revisited,” in Proc. of Pairing-Based Cryptography-Pairing 2009, pp. 114-131, 2009. Article (CrossRef Link)

[24] S. Brands, “Untraceable off-line cash in wallet with observers,” in Proc. of CRYPTO’93, pp. 302-318, 1993. Article (CrossRef Link)

[25] S. Brands and C. v. W. e. Informatica, “An efficient off-line electronic cash system based on the representation problem,” CWI Technical Report CS-R9323, Citeseer, 1970.

Article (CrossRef Link)

[26] S. Canard and A. Gouget, "Divisible e-cash systems can be truly anonymous," in Proc. of Advances in Cryptology-EUROCRYPT 2007, pp. 482-497, 2007. Article (CrossRef Link)

[27] Z. Eslami and M. Talebi, “A new untraceable off-line electronic cash system," Electronic Commerce Research and Applications, vol. 10, no. 1, pp. 59-66, 2011. Article (CrossRef Link)

[28] Schoenmakers, B., "Security aspects of the E-cash ${ }^{\mathrm{TM}}$ payment system," State of the Art in Applied Cryptography, pp. 338-352, 1998. Article (CrossRef Link)

[29] W. S. Juang, "RO-cash: An efficient and practical recoverable pre-paid offline e-cash scheme using bilinear pairings,” Journal of Systems and Software, vol. 83, pp. 638-645, 2010. Article (CrossRef Link)

[30] B. Lian, G. L. Chen and J. H. Li, "Provably secure E-cash system with practical and efficient complete tracing," International Journal of Information Security, vol. 13, no. 3, pp. 271-289, Apr. 2014. Article (CrossRef Link) 
[31] M. Au, Q Wu, W Susilo, Y Mu, “Compact E-Cash from Bounded Accumulator,” in Proc. of CT-RSA’07. LNCS, vol. 4377, pp. 178-195, 2007. Article (CrossRef Link)

[32] B. Lian, G. Chen, M. Ma, J. Li, "Periodic K-Times Anonymous Authentication with Efficient Revocation of Violator's Credential," IEEE Transactions on Information, Forensics and Security, VOL. 10, NO. 3, pp. 543-557. 2015. Article (CrossRef Link)

[33] E. Fujisaki and T. Okamoto, "Statistical zero knowledge protocols to prove modular polynomial relations,” in Proc. of Advances in Cryptology-CRYPTO'97, pp. 16-30, 1997. Article (CrossRef Link)

[34] D. Boneh, “The decision diffie-hellman problem,” Algorithmic Number Theory, pp. 48-63, 1998. Article (CrossRef Link)

[35] G. Ateniese, J. Camenisch, M. Joye, and G. Tsudik, “A practical and provably secure coalition-resistant group signature scheme,” in Proc. of Advances in Cryptology-CRYPTO 2000, pp. 255-270, 2000. Article (CrossRef Link)

[36] J. Camenisch, "Group signature schemes and payment systems based on the discrete logarithm problem," $\mathrm{PhD}$ thesis, vol. 2 of ETH Series in Information Security an Cryptography, Hartung-Gorre Verlag, Konstanz. ISBN 3-89649-286-1, 1998. Article (CrossRef Link)

[37] Y. Dodis and A. Yampolskiy, “A Verifiable Random Function With Short Proofs and Keys,” in Proc. of Public Key Cryptography, vol. 3386 of LNCS, pp. 416-431, 2005. Article (CrossRef Link)

[38] J. Camenisch and Anna Lysyanskaya, “A signature scheme with efficient protocols,” in Proc. of Security in Communication Networks'02, vol. 2576 of LNCS, pages 268-289. 2002. Article (CrossRef Link)

[39] J. Camenisch and M. Michels, "Proving in zero-knowledge that a number is the product of two safe primes,” in Proc. of Advances in Cryptology_EUROCRYPT’99, pp. 107-122, 1999. Article (CrossRef Link)

[40] C.P. Schnorr, "Efficient Signature Generation by Smart Cards,” Journal of Cryptology, vol 4, pp. 161-174, 1991. Article (CrossRef Link)

[41] B. Lian, GL. Chen, JH. Li, “ A Provably Secure and Practical Fair E-cash Scheme,” in Proc. of 2010 IEEE International Conference on Information Theory and Information Security, 2010. Article (CrossRef Link) 


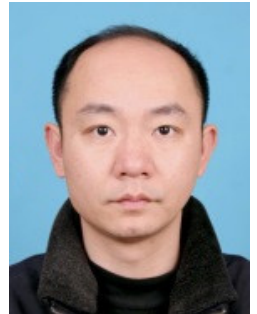

Bin Lian received the M.S. degree in cryptography from Southwest Jiaotong University in 2005 and the Ph.D. degree in cryptography from Shanghai Jiao Tong University in 2015. He is with the Ningbo Institute of Technology, Zhejiang University, Ningbo, China. His research interests include cryptography, cryptographic protocol and technology of network security.

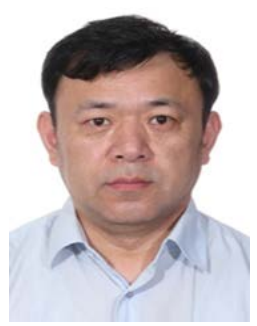

Gongliang Chen received his B.S. in Peking University, and M.S. degree in Chinese Academy of Science. In 1993, he received his Ph.D. degree in Université de Saint Etienne, France. He is also a visiting scholar of Université Paris VI, France. He is currently a professor at the School of Information Security Engineering, Shanghai Jiao Tong University, Shanghai. His main research area includes cryptographic theory and technology of network security.

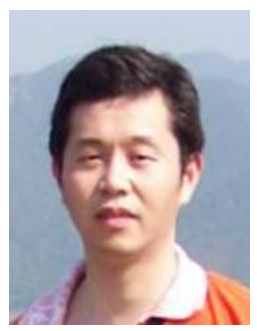

Jialin Cui received the M.S. degree from Zhejiang University in 2005. He is with the Ningbo Institute of Technology, Zhejiang University, Ningbo, China. He is currently pursuing the Ph.D. degree with Ningbo University. His research interests include cryptography, security protocol and cryptographic applications.

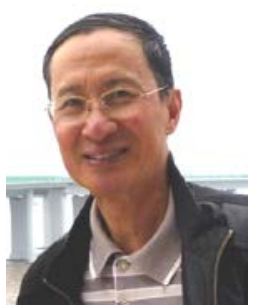

Dake He received his M.S. degree in Xidian University, Xian in 1981. He is a professor and doctoral supervisor at Southwest Jiaotong University, Chengdu. He is also the director of national high performance computing center (Chengdu). His research area includes cryptography, communications system security, security engineering of information system, parallel computing and applied mathematics. 